Supporting Information to Accompany

4'-Substituted-4-biphenylyloxenium Ions: Reactivity and Selectivity In Aqueous

Solution

Michael Novak, *Matthew J. Poturalski, Whitney L. Johnson, Matthew P. Jones, Yueting Wang, and Stephen A. Glover

Department of Chemistry and Biochemistry, Miami University, Oxford, OH 45056 USA

and

School of Biological, Biomedical, and Molecular Sciences, Division of Chemistry,

University of New England, Armidale, 2351, New South Wales, AUSTRALIA.

Contents

Page

Characterization of $\mathbf{4 b - f ,} 2 \mathbf{c}-\mathbf{e}, 2 \mathbf{e}^{\prime}$ and $2 \mathbf{f}^{\prime}$ S1

Synthesis of $\mathbf{3 b}$ and $\mathbf{3 c}$

S3

Characterization of $5 \mathbf{b}-\mathbf{f}$, and $\mathbf{6 b}$ :

S5

Z-matrices of the $\mathrm{HF} / 6-31 \mathrm{G}^{*}$ optimized $\mathrm{S} 6$ geometries of 1a-f, and 4a-f.

Comparisons of selected $\mathrm{HF} / 6-31 \mathrm{G}^{*}$ bond lengths $\mathrm{S} 14$ in 1a-f

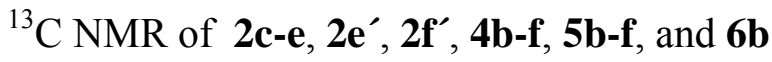

S15

Characterization of the 4-hydroxy-4-aryl-2,5-cyclohexadienones, 4b-f, the 4-acetoxy-4-aryl-2,5cyclohexadienones, 2c-e, and the 4-dichloroacetoxy-4-aryl-2,5-cyclohexadienones, $2 \mathrm{e}^{\prime}$ and 2f':

4-Hydroxy-4-(4'-methoxyphenyl)-2,5-cyclohexadienone (4b): mp 120-122 ${ }^{\circ} \mathrm{C}$; IR 3402, 1654, 1618, 1249, 1052, 1027, 860, $827 \mathrm{~cm}^{-1} ;{ }^{1} \mathrm{H}$ NMR (500 MHz, $\left.\mathrm{CD}_{2} \mathrm{Cl}_{2}\right) \delta 2.71(1 \mathrm{H}, \mathrm{s}), 3.83(3 \mathrm{H}, \mathrm{s}), 6.20(2 \mathrm{H}, \mathrm{d}$ $\mathrm{J}=10.1 \mathrm{~Hz}), 6.90-6.95(4 \mathrm{H}, \mathrm{m}), 7.42(2 \mathrm{H}, \mathrm{d} \mathrm{J}=8.9 \mathrm{~Hz}) ;{ }^{13} \mathrm{C} \mathrm{NMR}\left(125.8 \mathrm{MHz}, \mathrm{CD}_{2} \mathrm{Cl}_{2}\right) \delta 55.3,70.6$, $114.2,126.5,126.6,131.0,150.8,159.7,185.4$. 
4-Hydroxy-4-(4'-methylphenyl)-2,5-cyclohexadienone (4c): $\mathrm{mp} 134-137{ }^{\circ} \mathrm{C}$; IR 3352, 3174, 1659, 1611, 1047, $941 \mathrm{~cm}^{-1} ;{ }^{1} \mathrm{H}$ NMR $\left(300 \mathrm{MHz}, \mathrm{CDCl}_{3}\right) \delta 2.37(3 \mathrm{H}, \mathrm{s}), 6.24(2 \mathrm{H}, \mathrm{d} \mathrm{J}=10.0 \mathrm{~Hz}) 6.91(2 \mathrm{H}, \mathrm{d}$, $\mathrm{J}=10.0 \mathrm{~Hz}), 7.21(2 \mathrm{H}, \mathrm{d} \mathrm{J}=8.3 \mathrm{~Hz}) 7.38(2 \mathrm{H}, \mathrm{d} \mathrm{J}=8.3 \mathrm{~Hz}) ;{ }^{13} \mathrm{C} \mathrm{NMR}\left(75 \mathrm{MHz}, \mathrm{CDCl}_{3}\right) \delta 21.5,71.3$, $125.6,127.1,130.0,136.2,138.7,151.5,186.3$.

4-Hydroxy-4-(4'-bromophenyl)-2,5-cyclohexadienone (4d): $\mathrm{mp}$ 175-177 ${ }^{\circ} \mathrm{C}$; IR 3377,1664, 1613, 1059, 943, $824 \mathrm{~cm}^{-1} ;{ }^{1} \mathrm{H}$ NMR $\left(300 \mathrm{MHz}, \mathrm{CDCl}_{3}\right) \delta 1.6(1 \mathrm{H}, \mathrm{s}(\mathrm{br})), 6.26(2 \mathrm{H}, \mathrm{d} \mathrm{J}=9.9 \mathrm{~Hz}) 6.87(2 \mathrm{H}, \mathrm{d}$, $\mathrm{J}=10.0 \mathrm{~Hz}), 7.37(2 \mathrm{H}, \mathrm{d} \mathrm{J}=8.6 \mathrm{~Hz}) 7.52(2 \mathrm{H}, \mathrm{d} \mathrm{J}=8.6 \mathrm{~Hz}) ;{ }^{13} \mathrm{C} \mathrm{NMR}\left(75 \mathrm{MHz}, \mathrm{CDCl}_{3}\right) \delta 71.1,123.0$, $127.5,127.6,132.4,138.2,150.5,185.7$.

4-Hydroxy-4-(4'-cyanophenyl)-2,5-cyclohexadienone (4e): mp 173-174 ${ }^{\circ} \mathrm{C}$; IR 3367, 2228, 1664 , 1621, 1064, 948, $857 \mathrm{~cm}^{-1} ;{ }^{1} \mathrm{H}$ NMR $\left(300 \mathrm{MHz} \mathrm{CDCl}_{3}\right) \delta 1.6(1 \mathrm{H}, \mathrm{s}(\mathrm{br})), 6.31(2 \mathrm{H}, \mathrm{d} \mathrm{J}=10.0 \mathrm{~Hz}) 6.85$ $(2 \mathrm{H}, \mathrm{d}, \mathrm{J}=10.0 \mathrm{~Hz}), 7.63(2 \mathrm{H}, \mathrm{d} \mathrm{J}=8.6 \mathrm{~Hz}) 7.70(2 \mathrm{H}, \mathrm{d} \mathrm{J}=8.6 \mathrm{~Hz}) ;{ }^{13} \mathrm{C} \mathrm{NMR}\left(75 \mathrm{MHz}, \mathrm{CDCl}_{3}\right) \delta$ $71.2,112.7,118.7,126.7,128.1,133.1,144.3,149.8,185.4$.

4-Hydroxy-4-(4'-nitrophenyl)-2,5-cyclohexadienone (4f): $\mathrm{mp}$ 181-184 ${ }^{\circ} \mathrm{C}$; IR 3286, 2228, 1664, 1618, 1512, 1343, 1062, $857 \mathrm{~cm}^{-1} ;{ }^{1} \mathrm{H}$ NMR (300 MHz, $\left.\mathrm{CDCl}_{3}\right) \delta 1.6(1 \mathrm{H}, \mathrm{s}(\mathrm{br})), 6.33(2 \mathrm{H}, \mathrm{d} \mathrm{J}=10.1$ Hz) $6.87(2 \mathrm{H}, \mathrm{d}, \mathrm{J}=9.7 \mathrm{~Hz}), 7.69(2 \mathrm{H}, \mathrm{d} \mathrm{J}=8.9 \mathrm{~Hz}) 8.25(2 \mathrm{H}, \mathrm{d} \mathrm{J}=8.7 \mathrm{~Hz}) ;{ }^{13} \mathrm{C} \mathrm{NMR}(75 \mathrm{MHz}$, DMSO-d 6 ) $\delta 70.9,124.7,127.2,127.8,147.9,148.8,152.1,186.0$.

4-Acetoxy-4-(4'-methylphenyl)-2,5-cyclohexadienone (2c): mp 145-149 ${ }^{\circ} \mathrm{C}$; IR 1729, 1661, 1621, 1231, 1037, $983 \mathrm{~cm}^{-1} ;{ }^{1} \mathrm{H}$ NMR $\left(300 \mathrm{MHz}, \mathrm{CDCl}_{3}\right) \delta 2.20(3 \mathrm{H}, \mathrm{s}), 2.37(3 \mathrm{H}, \mathrm{s}), 6.34(2 \mathrm{H}, \mathrm{d} \mathrm{J}=10.2 \mathrm{~Hz})$ $6.98(2 \mathrm{H}, \mathrm{d}, \mathrm{J}=10.2 \mathrm{~Hz}), 7.21(2 \mathrm{H}, \mathrm{d} \mathrm{J}=8.4 \mathrm{~Hz}) 7.32(2 \mathrm{H}, \mathrm{d} \mathrm{J}=8.4 \mathrm{~Hz}) ;{ }^{13} \mathrm{C} \mathrm{NMR}(125.8 \mathrm{MHz}$, $\left.\mathrm{CD}_{2} \mathrm{Cl}_{2}\right) \delta 20.7,21.3,77.2,125.2,127.8,129.6,133.7,138.8,147.7,169.1,185.3$.

4-Acetoxy-4-(4'-bromophenyl)-2,5-cyclohexadienone (2d): mp 120-122 ${ }^{\circ} \mathrm{C}$; IR 1729, 1666, 1628, 1226, 1029, 984, $824 \mathrm{~cm}^{-1} ;{ }^{1} \mathrm{H}$ NMR $\left(300 \mathrm{MHz} \mathrm{CDCl}_{3}\right) \delta 2.21(3 \mathrm{H}, \mathrm{s}), 6.36(2 \mathrm{H}, \mathrm{d} \mathrm{J}=10.2 \mathrm{~Hz}) 6.94$ $(2 \mathrm{H}, \mathrm{d}, \mathrm{J}=10.1 \mathrm{~Hz}), 7.31(2 \mathrm{H}, \mathrm{d} \mathrm{J}=8.7 \mathrm{~Hz}) 7.53(2 \mathrm{H}, \mathrm{d} \mathrm{J}=8.7 \mathrm{~Hz}) ;{ }^{13} \mathrm{C} \mathrm{NMR}\left(75 \mathrm{MHz}, \mathrm{CD}_{2} \mathrm{Cl}_{2}\right) \delta$ 22.0, 77.6, 123.4, 128.0, 129.0, 132.8, 136.8, 147.8, 169.6, 185.8.

4-Acetoxy-4-(4'-cyanophenyl)-2,5-cyclohexadienone (2e): mp 90-96 ${ }^{\circ} \mathrm{C}$; IR 2233, 1734, 1666, 1628, 1234, 1039, 994, $829 \mathrm{~cm}^{-1} ;{ }^{1} \mathrm{H}$ NMR $\left(300 \mathrm{MHz}, \mathrm{CDCl}_{3}\right) \delta 2.24(3 \mathrm{H}, \mathrm{s}), 6.41(2 \mathrm{H}, \mathrm{d} \mathrm{J}=10.2 \mathrm{~Hz}) 6.94$ 
$(2 \mathrm{H}, \mathrm{d}, \mathrm{J}=10.2 \mathrm{~Hz}), 7.56(2 \mathrm{H}, \mathrm{d} \mathrm{J}=8.6 \mathrm{~Hz}) 7.70(2 \mathrm{H}, \mathrm{d} \mathrm{J}=8.6 \mathrm{~Hz}) ;{ }^{13} \mathrm{C} \mathrm{NMR}\left(75 \mathrm{MHz}, \mathrm{CD}_{2} \mathrm{Cl}_{2}\right) \delta$

$22.0,77.6,113.4,118.9,127.0,129.6,133.6,142.7,147.1,169.5,185.6$.

4-Dichloroacetoxy-4-(4'-cyanophenyl)-2,5-cyclohexadienone (2e'): mp 95-98 ${ }^{\circ} \mathrm{C}$; IR 2233, 1772 , 1669, 1630, 1161, $753 \mathrm{~cm}^{-1} ;{ }^{1} \mathrm{H}$ NMR $\left(300 \mathrm{MHz}, \mathrm{CDCl}_{3}\right) \delta 6.05(1 \mathrm{H}, \mathrm{s}), 6.48(2 \mathrm{H}, \mathrm{d} \mathrm{J}=10.1 \mathrm{~Hz}) 6.95$ $(2 \mathrm{H}, \mathrm{d}, \mathrm{J}=10.1 \mathrm{~Hz}), 7.61(2 \mathrm{H}, \mathrm{d} \mathrm{J}=8.6 \mathrm{~Hz}) 7.75(2 \mathrm{H}, \mathrm{d} \mathrm{J}=8.6 \mathrm{~Hz}) ;{ }^{13} \mathrm{C} \mathrm{NMR}\left(75 \mathrm{MHz}, \mathrm{CDCl}_{3}\right) \delta$ $64.5,79.5,113.8,118.2,126.4,130.5,133.5,141.1,144.2,162.2,184.5$

4-Dichloroacetoxy-4-(4'-nitrophenyl)-2,5-cyclohexadienone (2f'): orange oil; IR 1767, 1669, 1631, 1520, 1348, 1148, 991, $852 \mathrm{~cm}^{-1} ;{ }^{1} \mathrm{H}$ NMR $\left(300 \mathrm{MHz}, \mathrm{CDCl}_{3}\right) \delta 6.06(1 \mathrm{H}, \mathrm{s}), 6.50(2 \mathrm{H}, \mathrm{d} \mathrm{J}=10.2 \mathrm{~Hz})$ $6.98(2 \mathrm{H}, \mathrm{d}, \mathrm{J}=10.2 \mathrm{~Hz}), 7.68(2 \mathrm{H}, \mathrm{d} \mathrm{J}=9.0 \mathrm{~Hz}) 8.31(2 \mathrm{H}, \mathrm{d} \mathrm{J}=9.0 \mathrm{~Hz}) ;{ }^{13} \mathrm{C} \mathrm{NMR}\left(75 \mathrm{MHz}, \mathrm{CDCl}_{3}\right) \delta$ $64.5,79.4,124.9,126.7,130.6,142.9,144.0,148.7,162.2,184.4$.

\section{Synthesis of $\mathrm{O}$-(4-(4'-methoxyphenyl)phenyl)- $\mathrm{N}$-methanesulfonylhydroxylamine, $3 \mathrm{~b}$, and $\mathrm{O}$-(4-} (4'-methylphenyl)phenyl)- $N$-methanesulfonylhydroxylamine, 3c: These compounds were made from reaction of the hydroxylamines with methanesulfonyl chloride. ${ }^{12}$ The hydroxylamines were generated by amination of the 4-hydroxybiphenyls. ${ }^{14 b, 15}$

Hydroxylamines: ${ }^{15}$ The $4^{\prime}$-substituted-4-hydroxybiphenyl ${ }^{14 \mathrm{a}}(6.4 \mathrm{mmol})$ was dissolved in $13 \mathrm{~mL}$ of $\mathrm{MeOH}$. Powdered potassium tert-butoxide $(6.4 \mathrm{mmol})$ was added. After shaking, the stoppered solution was allowed to sit until it cooled to room temperature. The solvent was removed by rotary evaporation and the solid residue was kept under a vacuum of ca. 0.1 torr for $24 \mathrm{~h}$. The solid was then suspended in $9 \mathrm{~mL}$ of dry DMF, and stirred under $\mathrm{N}_{2}$ while it was cooled in an ice-water bath. A solution of $5.8 \mathrm{mmol}$ of freshly prepared mesitylenesolfonylhydroxylamine ${ }^{14 \mathrm{~b}}$ in $5 \mathrm{~mL}$ of dry DMF was added all at once from an addition funnel, and the resulting mixture was stirred in the ice-water bath for 30 min. The reaction mixture was then added to $100 \mathrm{~mL}$ of $\mathrm{H}_{2} \mathrm{O}$, and the suspension was extracted $(3 \times 50 \mathrm{~mL})$ with $\mathrm{CH}_{2} \mathrm{Cl}_{2}$. The combined organic extracts were dried over $\mathrm{Na}_{2} \mathrm{SO}_{4}$, filtered, and evaporated by rotary evaporation. Residual DMF was removed by subjecting the sample to a vacuum of ca. 0.1 torr overnight. The crude product was purified by column chromatography on silica gel $\left(\mathrm{CH}_{2} \mathrm{Cl}_{2}\right.$ eluent $)$. 
Yields for both hydroxylamines were 45-50\%. Unreacted 4-hydroxybiphenyls were recovered as the only other identifiable materials from the reaction.

O-(4-(4'-methoxyphenyl)phenyl)hydroxylamine: $\mathrm{mp} 131-132{ }^{\circ} \mathrm{C}$; IR $3318,1604,1493,1280,1237$, 1193, 1135, 1120, $1037 \mathrm{~cm}^{-1} ;{ }^{1} \mathrm{H}$ NMR (300 MHz, DMSO-d 6 ) $\delta 3.77(3 \mathrm{H}, \mathrm{s}), 6.92(2 \mathrm{H}, \mathrm{s}), 6.98(2 \mathrm{H}, \mathrm{d}, \mathrm{J}$ $=8.7 \mathrm{~Hz}), 7.11(2 \mathrm{H}, \mathrm{J}=8.9 \mathrm{~Hz}), 7.48-7.54(4 \mathrm{H}, \mathrm{m}) ;{ }^{13} \mathrm{C} \mathrm{NMR}\left(75 \mathrm{MHz}, \mathrm{DMSO}-\mathrm{d}_{6}\right) \delta$ 55.1, 113.4, $114.3,126.8,127.1,132.0,132.5,158.3,160.8$.

O-(4-(4'-methylyphenyl)phenyl)hydroxylamine: mp 114-115 ${ }^{\circ} \mathrm{C}$; IR 3318, 1606, 1494, 1244, 1136 , $1120 \mathrm{~cm}^{-1} ;{ }^{1} \mathrm{H}$ NMR (300 MHz, DMSO-d $) \delta 2.31(3 \mathrm{H}, \mathrm{s}), 6.93(2 \mathrm{H}, \mathrm{s}), 7.12(2 \mathrm{H}, \mathrm{d}, \mathrm{J}=8.8 \mathrm{~Hz}), 7.21$ $(2 \mathrm{H}, \mathrm{J}=8.0 \mathrm{~Hz}), 7.46-7.53(4 \mathrm{H}, \mathrm{m}) ;{ }^{13} \mathrm{C} \mathrm{NMR}\left(75 \mathrm{MHz}, \mathrm{DMSO}-\mathrm{d}_{6}\right) \delta 20.6,113.4,125.9,127.1,129.4$, $132.1,135.7,137.1,161.1$.

3b and 3c: ${ }^{12}$ The hydroxylamine $(0.5 \mathrm{mmol})$ was dissolved in $1 \mathrm{~mL}$ of dry pyridine, and the resulting solution was stirred under $\mathrm{N}_{2}$ while $54 \mu \mathrm{L}(0.7$ mmole $)$ of methanesulfonyl chloride was added via syringe. The mixture was stirred overnight at room temperature, diluted with $10 \mathrm{~mL}$ of $\mathrm{CH}_{2} \mathrm{Cl}_{2}$, and washed with $\mathrm{H}_{2} \mathrm{O}(2 \times 4 \mathrm{~mL})$ and brine $(2 \times 4 \mathrm{~mL})$. The organic solution was dried over $\mathrm{Na}_{2} \mathrm{SO}_{4}$, filtered, and concentrated on the rotary evaporator. Residual pyridine was removed by subjecting the sample to a vacuum of 0.1 torr overnight. The material that remained was then recrystallized from $\mathrm{CH}_{2} \mathrm{Cl}_{2}$. Yields of recrystallized product were typically ca. $50 \%$.

$O-\left(4-\left(4^{\prime}\right.\right.$-methoxyphenyl)phenyl)- $N$-methanesulfonylhydroxylamine (3b): mp $109-110{ }^{\circ} \mathrm{C}$; IR 3144 , 1601, 1489, 1312, $1143 \mathrm{~cm}^{-1} ;{ }^{1} \mathrm{H}$ NMR (500 MHz, DMSO-d $) \delta 3.18(3 \mathrm{H}, \mathrm{s}), 3.79,(3 \mathrm{H}, \mathrm{s}), 7.01(2 \mathrm{H}, \mathrm{d}$, $\mathrm{J}=8.8 \mathrm{~Hz}), 7.22(2 \mathrm{H}, \mathrm{d} \mathrm{J}=8.8 \mathrm{~Hz}), 7.55-7.58(4 \mathrm{H}, \mathrm{m}), 10.82(1 \mathrm{H}, \mathrm{s}) ;{ }^{13} \mathrm{C}$ NMR $(125.8 \mathrm{MHz}$, DMSO$\left.\mathrm{d}_{6}\right) \delta 37.5,55.6,114.8,115.1,127.6,127.9,132.5,135.1,159.1,159.2$.

$O-\left(4-\left(4^{\prime}\right.\right.$-methylphenyl)phenyl)- $N$-methanesulfonylhydroxylamine (3c): mp $114-116{ }^{\circ} \mathrm{C}$; IR 3144, 1601, 1489, 1312, $1146 \mathrm{~cm}^{-1} ;{ }^{1} \mathrm{H}$ NMR (300 MHz, DMSO-d $) \delta 2.33(3 \mathrm{H}, \mathrm{s}), 3.18,(3 \mathrm{H}, \mathrm{s}), 7.21-7.26$ $(4 \mathrm{H}, \mathrm{m}), 7.52(2 \mathrm{H}, \mathrm{d} \mathrm{J}=8.0 \mathrm{~Hz}), 7.61(2 \mathrm{H}, \mathrm{d}, \mathrm{J}=8.6 \mathrm{~Hz}), 10.83(1 \mathrm{H}, \mathrm{s}) ;{ }^{13} \mathrm{C} \mathrm{NMR}(75 \mathrm{MHz}$, DMSO$\left.\mathrm{d}_{6}\right) \delta 21.5,37.9,115.4,127.1,128.3,130.4,135.7,137.1,137.5,159.8$. 
Characterization of the azide adducts, $5 b-f$, and the bromo adduct, $6 \mathbf{b}:{ }^{13} \mathrm{C}$ NMR assignments were made from HSQC and HMBC experiments.

3-Azido-4-hydroxy-4'-methoxybiphenyl (5b): mp 103-104 ${ }^{\circ} \mathrm{C}$; IR 3403, 2106, 1608, 1492, 1244, 814 $\mathrm{cm}^{-1} ;{ }^{1} \mathrm{H}$ NMR $\left(500 \mathrm{MHz}, \mathrm{CD}_{2} \mathrm{Cl}_{2}\right) \delta 3.87(3 \mathrm{H}, \mathrm{s}), 5.43(1 \mathrm{H}, \mathrm{s}(\mathrm{br})), 6.99(1 \mathrm{H}, \mathrm{d}, \mathrm{J}=8.2 \mathrm{~Hz}), 7.00(2 \mathrm{H} \mathrm{d}$, $\mathrm{J}=8.8 \mathrm{~Hz}) 7.28(1 \mathrm{H} \mathrm{dd}, \mathrm{J}=2.1,8.3 \mathrm{~Hz}), 7.31(1 \mathrm{H}, \mathrm{d}, \mathrm{J}=2.0 \mathrm{~Hz}), 7.52(2 \mathrm{H}, \mathrm{d}, \mathrm{J}=8.8 \mathrm{~Hz}) ;{ }^{13} \mathrm{C} \mathrm{NMR}$ $\left(75 \mathrm{MHz}, \mathrm{CD}_{2} \mathrm{Cl}_{2}\right) \delta 55.7(\mathrm{MeO}), 114.6\left(\mathrm{C}-3^{\prime}, \mathrm{C}-5^{\prime}\right), 116.5(\mathrm{C}-5), 116.9(\mathrm{C}-2), 124.7(\mathrm{C}-6), 126.7(\mathrm{C}-1)$, 128.0(C-2',C-4'), 132.8(C-1'), 134.7(C-3), 146.8(C-4), 159.6(C-4');

3-Azido-4-hydroxy-4'-methylbiphenyl (5c): mp 97-98 C; IR 3397, 2101, 1596, 1497, 1211, $802 \mathrm{~cm}^{-}$ ${ }^{1} ;{ }^{1} \mathrm{H}$ NMR $\left(500 \mathrm{MHz}, \mathrm{CD}_{2} \mathrm{Cl}_{2}\right) \delta 2.42(3 \mathrm{H}, \mathrm{s}), 5.41(1 \mathrm{H}, \mathrm{s}(\mathrm{br})), 7.00(1 \mathrm{H}, \mathrm{d}, \mathrm{J}=8.3 \mathrm{~Hz}), 7.28(2 \mathrm{H} \mathrm{d}, \mathrm{J}=$ $8.0 \mathrm{~Hz}) 7.32(1 \mathrm{H}, \mathrm{dd}, \mathrm{J}=2.1,8.3 \mathrm{~Hz}), 7.34(1 \mathrm{H}, \mathrm{d}, \mathrm{J}=2.1 \mathrm{~Hz}), 7.48(2 \mathrm{H}, \mathrm{d}, \mathrm{J}=8.0 \mathrm{~Hz}) ;{ }^{13} \mathrm{C}$ NMR $(75$ $\left.\mathrm{MHz}, \mathrm{CD}_{2} \mathrm{Cl}_{2}\right) \delta$ 21.1(Me), 116.5(C-5), 117.2(C-2), 124.9(C-6), 126.7(C-1), 126.8(C-2',C-6'), 129.9(C$\left.3^{\prime}, \mathrm{C}-5^{\prime}\right), 135.0\left(\mathrm{C}-1^{\prime}\right), 137.4(\mathrm{C}-3), 137.5\left(\mathrm{C}-4^{\prime}\right), 147.0(\mathrm{C}-4)$;

3-Azido-4-hydroxy-4'-bromobiphenyl (5d): mp 93-94 ${ }^{\circ} \mathrm{C}$; IR 3372, 2101, 1596, 1484, 1219, $804 \mathrm{~cm}^{-}$ ${ }^{1} ;{ }^{1} \mathrm{H}$ NMR $\left(500 \mathrm{MHz}, \mathrm{CD}_{2} \mathrm{Cl}_{2}\right) \delta 5.52(1 \mathrm{H}, \mathrm{s}(\mathrm{br})), 7.01(1 \mathrm{H}, \mathrm{d}, \mathrm{J}=8.2 \mathrm{~Hz}), 7.31(1 \mathrm{H} \mathrm{dd}, \mathrm{J}=2.1,8.2$ Hz) $7.33(1 \mathrm{H}, \mathrm{d}, \mathrm{J}=1.9 \mathrm{~Hz}), 7.48(2 \mathrm{H}, \mathrm{d}, \mathrm{J}=8.6 \mathrm{~Hz}), 7.59(2 \mathrm{H}, \mathrm{d}, \mathrm{J}=8.6 \mathrm{~Hz}) ;{ }^{13} \mathrm{C} \mathrm{NMR}(75 \mathrm{MHz}$, $\left.\mathrm{CD}_{2} \mathrm{Cl}_{2}\right) \delta 116.7(\mathrm{C}-5), 117.2(\mathrm{C}-2), 121.6\left(\mathrm{C}-4^{\prime}\right), 125.0(\mathrm{C}-6), 127.0(\mathrm{C}-1), 128.7\left(\mathrm{C}-2^{\prime}, \mathrm{C}-6^{\prime}\right), 132.3(\mathrm{C}-$ $\left.3^{\prime}, \mathrm{C}-5^{\prime}\right), 133.7(\mathrm{C}-3), 139.3\left(\mathrm{C}-1^{\prime}\right), 147.6(\mathrm{C}-4)$;

3-Azido-4-hydroxy-4'-cyanobiphenyl (5e): mp 145.5-146.5 ${ }^{\circ} \mathrm{C}$; IR 3327, 2238, 2106, 1603, 1497, 1262, $804 \mathrm{~cm}^{-1} ;{ }^{1} \mathrm{H}$ NMR $\left(500 \mathrm{MHz}, \mathrm{CDCl}_{3}\right) \delta 5.5(1 \mathrm{H}, \mathrm{s}(\mathrm{br})), 7.06(1 \mathrm{H}, \mathrm{d}, \mathrm{J}=8.2 \mathrm{~Hz}), 7.30(1 \mathrm{H} \mathrm{d}, \mathrm{J}=$ $2.0 \mathrm{~Hz}) 7.32(1 \mathrm{H}, \mathrm{dd}, \mathrm{J}=2.0,8.2 \mathrm{~Hz}), 7.66(2 \mathrm{H}, \mathrm{d}, \mathrm{J}=8.5 \mathrm{~Hz}), 7.75(2 \mathrm{H}, \mathrm{d}, \mathrm{J}=8.4 \mathrm{~Hz}) ;{ }^{13} \mathrm{C}$ NMR $(75$ $\left.\mathrm{MHz}, \mathrm{CDCl}_{3}\right) \delta 111.2\left(\mathrm{C}-4^{\prime}\right), 117.0(\mathrm{C}-5), 117.3(\mathrm{C}-2), 119.2(\mathrm{CN}), 125.5(\mathrm{C}-6), 127.2(\mathrm{C}-1), 127.7(\mathrm{C}-$ $\left.2^{\prime}, \mathrm{C}-6^{\prime}\right), 133.0(\mathrm{C}-3), 133.1\left(\mathrm{C}-3^{\prime}, \mathrm{C}-5^{\prime}\right), 144.8\left(\mathrm{C}-1^{\prime}\right), 147.6(\mathrm{C}-4)$;

3-Azido-4-hydroxy-4'-nitrobiphenyl (5f): mp 145-146 ${ }^{\circ} \mathrm{C}$; IR 3469, 2113, 1589, 1517, 1263, 1207 $\mathrm{cm}^{-1} ;{ }^{1} \mathrm{H}$ NMR $\left(500 \mathrm{MHz}, \mathrm{CDCl}_{3}\right) \delta 5.51(1 \mathrm{H}, \mathrm{s}(\mathrm{br})), 7.07(1 \mathrm{H}, \mathrm{d}, \mathrm{J}=8.3 \mathrm{~Hz}), 7.34(1 \mathrm{H}, \mathrm{d}, \mathrm{J}=2.0 \mathrm{~Hz})$, $7.36(1 \mathrm{H}, \mathrm{dd}, \mathrm{J}=1.9,8.3 \mathrm{~Hz}), 7.70(2 \mathrm{H}, \mathrm{d}, \mathrm{J}=8.9 \mathrm{~Hz}), 8.31(2 \mathrm{H}, \mathrm{d}, \mathrm{J}=8.9 \mathrm{~Hz}) ;{ }^{13} \mathrm{C} \mathrm{NMR}(75 \mathrm{MHz}$, 
$\left.\mathrm{CDCl}_{3}\right) \delta \quad 117.1(\mathrm{C}-5), \quad 117.5(\mathrm{C}-2), \quad 124.6\left(\mathrm{C}^{\prime} 3^{\prime}, \mathrm{C}-5^{\prime}\right), \quad 125.7(\mathrm{C}-6), \quad 127.3(\mathrm{C}-1), \quad 127.7\left(\mathrm{C}-2^{\prime}, \mathrm{C}^{\prime} 6^{\prime}\right)$, 132.6(C-3), 146.7(C-1'), 147.4(C-4'), 148.5(C-4);

3-bromo-4-hydroxy-4'-methoxybiphenyl (6b): mp 85-86.5 ${ }^{\circ} \mathrm{C}$; IR cm ${ }^{-1} ;{ }^{1} \mathrm{H}$ NMR (500 MHz, $\mathrm{CD}_{2} \mathrm{Cl}_{2}$ ) $\delta 3.87(3 \mathrm{H}, \mathrm{s}), 5.62(1 \mathrm{H}, \mathrm{s}(\mathrm{br})), 7.00(2 \mathrm{H}, \mathrm{d}, \mathrm{J}=8.7 \mathrm{~Hz}), 7.09(1 \mathrm{H} \mathrm{d}, \mathrm{J}=8.5 \mathrm{~Hz}), 7.46(1 \mathrm{H}, \mathrm{dd}, \mathrm{J}=2.2$,

$8.4 \mathrm{~Hz}), 7.50(2 \mathrm{H}, \mathrm{d}, \mathrm{J}=8.7 \mathrm{~Hz}), 7.72(1 \mathrm{H}, \mathrm{d}, \mathrm{J}=2.2 \mathrm{~Hz}) ;{ }^{13} \mathrm{C} \mathrm{NMR}\left(125.7 \mathrm{MHz}, \mathrm{CD}_{2} \mathrm{Cl}_{2}\right) \delta$ 55.3(MeO), 110.5(C-3), 114.2(C-3',C-5'), 116.2(C-5), 127.4(C-6), 127.6(C-2',C-6'), 130.0(C-2), 131.8(C-1), 135.0(C-1'), 151.3(C-4), 159.2(C-4');

\section{Z-matrices of the HF/6-31G* optimized geometries for oxenium ions, 1a-f, and their hydration products, 4a-f.}

Energies in a.u. (Hartree)

4-Biphenylyloxenium (1a)

22

$\begin{array}{lllllllll}\mathrm{C} & 1 & 0.000000 & 0.649857 & -0.473389 & 1 & 2 & 13 & 17\end{array}$

$\begin{array}{lllllllll}\mathrm{C} & 2 & 0.000000 & -0.499146 & 0.362656 & 1 & 1 & 3 & 7\end{array}$

$\begin{array}{lllllllll}\text { C } & 3 & -1.248993 & -1.111938 & 0.805405 & 1 & 2 & 4 & 12\end{array}$

$\begin{array}{lllllllll}\text { C } & 4 & -1.260986 & -2.189545 & 1.588348 & 1 & 3 & 5 & 11\end{array}$

$\begin{array}{lllllllll}\text { C } & 5 & 0.000000 & -2.823441 & 2.051392 & 1 & 4 & 6 & 10\end{array}$

$\begin{array}{lllllllll}\text { C } & 6 & 1.260986 & -2.185806 & 1.591812 & 1 & 5 & 7 & 9\end{array}$

$\begin{array}{lllllllll}\text { C } & 7 & 1.248993 & -1.109589 & 0.808640 & 1 & 2 & 6 & 8\end{array}$

$\begin{array}{lllllll}\mathrm{H} & 8 & 2.181000 & -0.686707 & 0.502640 & 5 & 7\end{array}$

$\begin{array}{lllllll}\mathrm{H} & 9 & 2.176987 & -2.637894 & 1.923996 & 5 & 6\end{array}$

$\begin{array}{lllllll}\mathrm{O} & 10 & 0.000000 & -3.783981 & 2.750488 & 6 & 5\end{array}$

$\begin{array}{lllllll}\mathrm{H} & 11 & -2.176987 & -2.644348 & 1.915100 & 5 & 4\end{array}$

$\begin{array}{lllllll}\mathrm{H} & 12 & -2.181000 & -0.690216 & 0.497787 & 5 & 3\end{array}$

$\begin{array}{lllllllll}\text { C } & 13 & 1.213989 & 1.248978 & -0.911148 & 1 & 1 & 14 & 22\end{array}$

$\begin{array}{lllllllll}\text { C } & 14 & 1.211000 & 2.357086 & -1.715027 & 1 & 13 & 15 & 21\end{array}$

$\begin{array}{lllllllll}\text { C } & 15 & 0.000000 & 2.914871 & -2.117813 & 1 & 14 & 16 & 20\end{array}$

$\begin{array}{lllllllll}\text { C } & 16 & -1.211000 & 2.360245 & -1.712372 & 1 & 15 & 17 & 19\end{array}$

$\begin{array}{lllllllll}\text { C } & 17 & -1.213989 & 1.251907 & -0.907104 & 1 & 1 & 16 & 18\end{array}$

$\begin{array}{lllllll}\mathrm{H} & 18 & -2.161987 & 0.853195 & -0.613724 & 5 & 17\end{array}$

$\begin{array}{lllllll}\mathrm{H} & 19 & -2.138992 & 2.798294 & -2.028168 & 5 & 16\end{array}$

$\begin{array}{lllllll}\mathrm{H} & 20 & 0.000000 & 3.785141 & -2.748871 & 5 & 15\end{array}$

$\begin{array}{lllllll}\mathrm{H} & 21 & 2.138992 & 2.793365 & -2.033234 & 5 & 14\end{array}$

$\begin{array}{lllllll}\mathrm{H} & 22 & 2.161987 & 0.846741 & -0.622620 & 5 & 13\end{array}$

\section{$\mathrm{E}\left(\mathrm{HF} / 6-31 \mathrm{G}^{*}\right) \quad-534.252124$ \\ $\mathrm{E}(\mathrm{pBP} / \mathrm{DN} * / / \mathrm{HF} / 6-$ \\ $31 \mathrm{G}^{*}$ \\ $-537.76495$ \\ $\mathrm{E}\left(\mathrm{B} 3 \mathrm{LYP} / 6-31 \mathrm{G}^{*}\right) \quad-537.616594$ \\ $\mathrm{E}\left(\mathrm{BP} / 6-31 \mathrm{G}^{*}\right) \quad-537.60196$}

4'-Methoxy-4-biphenylyloxenium (1b) 


\begin{tabular}{|c|c|c|c|c|c|c|c|c|}
\hline $\mathrm{C}$ & 1 & 0.287491 & -0.095993 & 0.000000 & 1 & 2 & 13 & 17 \\
\hline $\mathrm{C}$ & 2 & 1.674484 & 0.053009 & 0.000000 & 1 & 1 & 3 & 7 \\
\hline $\mathrm{C}$ & 3 & 2.308487 & 1.374985 & 0.000000 & 1 & 2 & 4 & 12 \\
\hline $\mathrm{C}$ & 4 & 3.628479 & 1.521988 & 0.000000 & 1 & 3 & 5 & 11 \\
\hline $\mathrm{C}$ & 5 & 4.542480 & 0.351990 & 0.000000 & 1 & 4 & 6 & 10 \\
\hline $\mathrm{C}$ & 6 & 3.890488 & -0.981995 & 0.000000 & 1 & 5 & 7 & 9 \\
\hline $\mathrm{C}$ & 7 & 2.567490 & -1.110000 & 0.000000 & 1 & 2 & 6 & 8 \\
\hline $\mathrm{H}$ & 8 & 2.148483 & -2.092987 & 0.000000 & 5 & 7 & & \\
\hline $\mathrm{H}$ & 9 & 4.543488 & -1.834000 & 0.000000 & 5 & 6 & & \\
\hline $\mathrm{O}$ & 10 & 5.724487 & 0.475998 & 0.000000 & 6 & 5 & & \\
\hline $\mathrm{H}$ & 11 & 4.091492 & 2.491989 & 0.000000 & 5 & 4 & & \\
\hline $\mathrm{H}$ & 12 & 1.696487 & 2.251984 & 0.000000 & 5 & 3 & & \\
\hline $\mathrm{C}$ & 13 & -0.348495 & -1.386993 & 0.000000 & 1 & 1 & 14 & 21 \\
\hline $\mathrm{C}$ & 14 & -1.691498 & -1.526993 & 0.000000 & 1 & 13 & 15 & 20 \\
\hline $\mathrm{C}$ & 15 & -2.527496 & -0.380997 & 0.000000 & 1 & 14 & 16 & 22 \\
\hline $\mathrm{C}$ & 16 & -1.948502 & 0.908997 & 0.000000 & 1 & 15 & 17 & 19 \\
\hline $\mathrm{C}$ & 17 & -0.596497 & 1.033997 & 0.000000 & 1 & 1 & 16 & 18 \\
\hline $\mathrm{H}$ & 18 & -0.202500 & 2.027985 & 0.000000 & 5 & 17 & & \\
\hline $\mathrm{H}$ & 19 & -2.564500 & 1.784988 & 0.000000 & 5 & 16 & & \\
\hline $\mathrm{H}$ & 20 & -2.159500 & -2.491989 & 0.000000 & 5 & 14 & & \\
\hline $\mathrm{H}$ & 21 & 0.237488 & -2.280991 & 0.000000 & 5 & 13 & & \\
\hline $\mathrm{O}$ & 22 & -3.797500 & -0.612000 & 0.000000 & 6 & 15 & 23 & \\
\hline $\mathrm{C}$ & 23 & -4.775497 & 0.428986 & 0.000000 & 1 & 22 & 24 & 25 \\
\hline $\mathrm{H}$ & 24 & -4.677505 & 1.031998 & -0.890991 & 5 & 23 & & \\
\hline $\mathrm{H}$ & 25 & -4.677505 & 1.031998 & 0.890991 & 5 & 23 & & \\
\hline $\mathrm{H}$ & 26 & -5.724503 & -0.077988 & 0.000000 & 5 & 23 & & \\
\hline \multirow{2}{*}{\multicolumn{3}{|c|}{$\begin{array}{l}\mathrm{E}\left(\mathrm{HF} / 6-31 \mathrm{G}^{*}\right) \\
\mathrm{E}(\mathrm{pBP} / \mathrm{DN} * / / \mathrm{HF} / 6-\end{array}$}} & \multicolumn{2}{|c|}{-648.1510145} & & & & \\
\hline & & & & & & & & \\
\hline \multicolumn{2}{|c|}{$31 \mathrm{G}^{*}$} & & \multicolumn{2}{|c|}{-652.352151} & & & & \\
\hline \multicolumn{3}{|c|}{$\mathrm{E}\left(\mathrm{B} 3 \mathrm{LYP} / 6-31 \mathrm{G}^{*}\right)$} & \multicolumn{2}{|c|}{-652.158197} & & & & \\
\hline \multicolumn{3}{|c|}{ E(BP/6-31G*) } & \multicolumn{2}{|c|}{-652.145624} & & & & \\
\hline
\end{tabular}

4'-Methyl-4-biphenylyloxenium (1c)
$\begin{array}{ccccccccc}\text { 25 } & & & & & & \\ \text { C } & 1 & 0.352692 & -0.003006 & 0.101044 & 1 & 2 & 13 & 17 \\ \text { C } & 2 & -1.027252 & 0.000000 & -0.193298 & 1 & 1 & 3 & 7 \\ \text { C } & 3 & -1.768188 & 1.250992 & -0.343643 & 1 & 2 & 4 & 12 \\ \text { C } & 4 & -3.070313 & 1.264984 & -0.619400 & 1 & 3 & 5 & 11 \\ \text { C } & 5 & -3.841232 & 0.006989 & -0.787354 & 1 & 4 & 6 & 10 \\ \text { C } & 6 & -3.073486 & -1.255005 & -0.633347 & 1 & 5 & 7 & 9 \\ \text { C } & 7 & -1.772339 & -1.248000 & -0.357803 & 1 & 2 & 6 & 8 \\ \text { H } & 8 & -1.263016 & -2.182007 & -0.257721 & 5 & 7 & & \\ \text { H } & 9 & -3.623138 & -2.169998 & -0.755295 & 5 & 6 & & \\ \text { O } & 10 & -5.003891 & 0.008987 & -1.031418 & 6 & 5 & & \\ \text { H } & 11 & -3.618347 & 2.181992 & -0.729752 & 5 & 4 & & \\ \text { H } & 12 & -1.257660 & 2.181992 & -0.230011 & 5 & 3 & & \\ \text { C } & 13 & 1.083100 & -1.216003 & 0.262421 & 1 & 1 & 14 & 22 \\ \text { C } & 14 & 2.418839 & -1.216003 & 0.548386 & 1 & 13 & 15 & 21 \\ \text { C } & 15 & 3.120987 & -0.013000 & 0.693558 & 1 & 14 & 16 & 20 \\ \text { C } & 16 & 2.425797 & 1.197983 & 0.539658 & 1 & 15 & 17 & 19\end{array}$




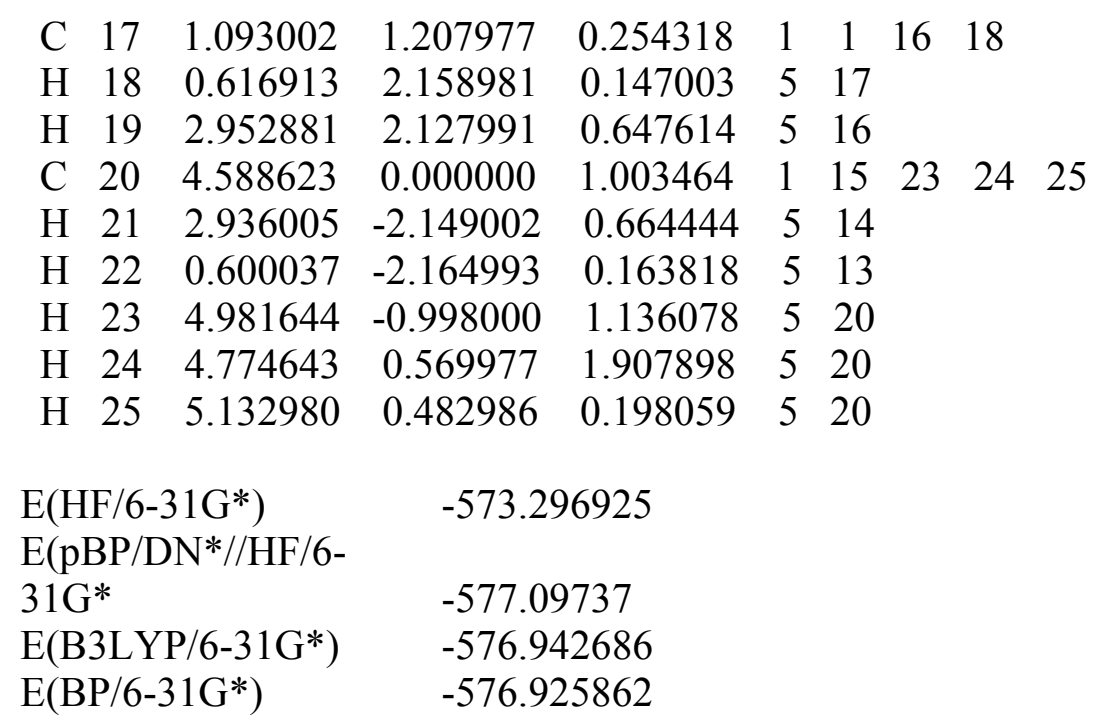

4'-Bromo-4-biphenylyloxenium (1d)

22

$\begin{array}{lllllllll}\text { C } & 1 & 0.000000 & -0.405121 & -0.017685 & 1 & 2 & 13 & 17\end{array}$

$\begin{array}{lllllllll}\mathrm{C} & 2 & 0.000000 & 1.012527 & 0.044205 & 1 & 1 & 3 & 7\end{array}$

$\begin{array}{lllllllll}\text { C } & 3 & 1.248993 & 1.766815 & 0.077118 & 1 & 2 & 4 & 12\end{array}$

$\begin{array}{lllllllll}\text { C } & 4 & 1.260986 & 3.097549 & 0.135208 & 1 & 3 & 5 & 11\end{array}$

$\begin{array}{lllllllll}\text { C } & 5 & 0.000000 & 3.882797 & 0.169495 & 1 & 4 & 6 & 10\end{array}$

$\begin{array}{lllllllll}\text { C } & 6 & -1.260986 & 3.097549 & 0.135208 & 1 & 5 & 7 & 9\end{array}$

$\begin{array}{lllllllll}\text { C } & 7 & -1.248993 & 1.766815 & 0.077118 & 1 & 2 & 6 & 8\end{array}$

$\begin{array}{lllllll}\mathrm{H} & 8 & -2.181000 & 1.246292 & 0.054398 & 5 & 7\end{array}$

$\begin{array}{lllllll}\mathrm{H} & 9 & -2.176987 & 3.658005 & 0.159683 & 5 & 6\end{array}$

$\begin{array}{lllllll}\mathrm{O} & 10 & 0.000000 & 5.068665 & 0.221252 & 6 & 5\end{array}$

$\begin{array}{lllllll}\mathrm{H} & 11 & 2.176987 & 3.658005 & 0.159683 & 5 & 4\end{array}$

$\begin{array}{lllllll}\mathrm{H} & 12 & 2.181000 & 1.246292 & 0.054398 & 5 & 3\end{array}$

$\begin{array}{lllllllll}\text { C } & 13 & -1.211000 & -1.150391 & -0.050217 & 1 & 1 & 14 & 22\end{array}$

$\begin{array}{lllllllll}\text { C } & 14 & -1.214996 & -2.516098 & -0.109833 & 1 & 13 & 15 & 21\end{array}$

$\begin{array}{lllllllll}\text { C } & 15 & 0.000000 & -3.203445 & -0.139832 & 1 & 14 & 16 & 20\end{array}$

$\begin{array}{lllllllll}\text { C } & 16 & 1.214996 & -2.516098 & -0.109833 & 1 & 15 & 17 & 19\end{array}$

$\begin{array}{lllllllll}\text { C } & 17 & 1.211000 & -1.150391 & -0.050217 & 1 & 1 & 16 & 18\end{array}$

$\begin{array}{lllllll}\mathrm{H} & 18 & 2.161987 & -0.660873 & -0.028854 & 5 & 17\end{array}$

$\begin{array}{lllllll}\mathrm{H} & 19 & 2.140000 & -3.056580 & -0.133423 & 5 & 16\end{array}$

$\begin{array}{lllllll}\mathrm{Br} & 20 & 0.000000 & -5.068680 & -0.221252 & 13 & 15\end{array}$

$\begin{array}{lllllll}\mathrm{H} & 21 & -2.140000 & -3.056580 & -0.133423 & 5 & 14\end{array}$

$\begin{array}{lllllll}\mathrm{H} & 22 & -2.161987 & -0.660873 & -0.028854 & 5 & 13\end{array}$

$\begin{array}{ll}\mathrm{E}\left(\mathrm{HF} / 6-31 \mathrm{G}^{*}\right) & -3105.767404 \\ \mathrm{E}\left(\mathrm{pBP} / \mathrm{DN}{ }^{*} / \mathrm{HF} / 6-\right. & \\ 31 \mathrm{G}^{*} & -3111.667331 \\ \mathrm{E}\left(\mathrm{B} 3 \mathrm{LYP} / 6-31 \mathrm{G}^{*}\right) & -3110.8961 \\ \mathrm{E}\left(\mathrm{BP} / 6-31 \mathrm{G}^{*}\right) & -3111.13772\end{array}$

4'-Cyano-4-biphenylyloxenium (1e)

23

$\begin{array}{lllllllll}\mathrm{C} & 1 & 0.000000 & -0.034698 & 0.045227 & 1 & 2 & 13 & 17\end{array}$

$\begin{array}{lllllllll}\mathrm{C} & 2 & 0.000000 & 0.837631 & -1.091644 & 1 & 1 & 3 & 7\end{array}$

$\begin{array}{lllllllll}\text { C } & 3 & 1.248993 & 1.292984 & -1.685074 & 1 & 2 & 4 & 12\end{array}$ 


$\begin{array}{ccccccccc}\mathrm{C} & 4 & 1.261993 & 2.105072 & -2.743408 & 1 & 3 & 5 & 11 \\ \mathrm{C} & 5 & 0.000000 & 2.582336 & -3.365387 & 1 & 4 & 6 & 10 \\ \mathrm{C} & 6 & -1.261993 & 2.105072 & -2.743408 & 1 & 5 & 7 & 9 \\ \mathrm{C} & 7 & -1.248993 & 1.292984 & -1.685074 & 1 & 2 & 6 & 8 \\ \mathrm{H} & 8 & -2.179993 & 0.974609 & -1.270142 & 5 & 7 & & \\ \mathrm{H} & 9 & -2.177994 & 2.445984 & -3.187683 & 5 & 6 & & \\ \mathrm{O} & 10 & 0.000000 & 3.304947 & -4.307114 & 6 & 5 & & \\ \mathrm{H} & 11 & 2.177994 & 2.445984 & -3.187683 & 5 & 4 & & \\ \mathrm{H} & 12 & 2.179993 & 0.974609 & -1.270142 & 5 & 3 & & \\ \mathrm{C} & 13 & -1.211000 & -0.483353 & 0.629929 & 1 & 1 & 14 & 21 \\ \mathrm{C} & 14 & -1.212997 & -1.317963 & 1.717621 & 1 & 13 & 15 & 20 \\ \mathrm{C} & 15 & 0.000000 & -1.736800 & 2.263458 & 1 & 14 & 16 & 22 \\ \mathrm{C} & 16 & 1.212997 & -1.317963 & 1.717621 & 1 & 15 & 17 & 19 \\ \mathrm{C} & 17 & 1.211000 & -0.483353 & 0.629929 & 1 & 1 & 16 & 18 \\ \mathrm{H} & 18 & 2.159988 & -0.184464 & 0.240387 & 5 & 17 & & \\ \mathrm{H} & 19 & 2.137985 & -1.648529 & 2.148407 & 5 & 16 & & \\ \mathrm{H} & 20 & -2.137985 & -1.648529 & 2.148407 & 5 & 14 & & \\ \mathrm{H} & 21 & -2.159988 & -0.184464 & 0.240387 & 5 & 13 & & \\ \mathrm{C} & 22 & 0.000000 & -2.614014 & 3.406677 & 1 & 15 & 23 & \\ \mathrm{~N} & 23 & 0.000000 & -3.304947 & 4.307129 & 8 & 22 & & \end{array}$

$\begin{array}{ll}\mathrm{E}\left(\mathrm{HF} / 6-31 \mathrm{G}^{*}\right) & -625.9669434 \\ \mathrm{E}(\mathrm{pBP} / \mathrm{DN} / / \mathrm{HF} / 6- & \\ 31 \mathrm{G}^{*} & -630.025044 \\ \mathrm{E}\left(\mathrm{B} 3 \mathrm{LYP} / 6-31 \mathrm{G}^{*}\right) & -629.841381 \\ \mathrm{E}\left(\mathrm{BP} / 6-31 \mathrm{G}^{*}\right) & -629.829252\end{array}$

4'-Nitro-4-biphenylyloxenium (1f)

24

$\begin{array}{lrrrrrrrr}\mathrm{C} & 1 & 0.000000 & -0.304871 & 0.190506 & 1 & 2 & 13 & 17 \\ \mathrm{C} & 2 & 0.000000 & 0.916306 & -0.572571 & 1 & 1 & 3 & 7 \\ \mathrm{C} & 3 & 1.248993 & 1.548111 & -0.967361 & 1 & 2 & 4 & 12 \\ \mathrm{C} & 4 & 1.261993 & 2.679413 & -1.674271 & 1 & 3 & 5 & 11 \\ \mathrm{C} & 5 & 0.000000 & 3.345123 & -2.090256 & 1 & 4 & 6 & 10 \\ \mathrm{C} & 6 & -1.261993 & 2.679413 & -1.674271 & 1 & 5 & 7 & 9 \\ \mathrm{C} & 7 & -1.248993 & 1.548111 & -0.967361 & 1 & 2 & 6 & 8 \\ \mathrm{H} & 8 & -2.178986 & 1.103729 & -0.689682 & 5 & 7 & & \\ \mathrm{H} & 9 & -2.178986 & 3.155167 & -1.971558 & 5 & 6 & & \\ \mathrm{O} & 10 & 0.000000 & 4.350906 & -2.718735 & 6 & 5 & & \\ \mathrm{H} & 11 & 2.178986 & 3.155167 & -1.971558 & 5 & 4 & & \\ \mathrm{H} & 12 & 2.178986 & 1.103729 & -0.689682 & 5 & 3 & & \\ \mathrm{C} & 13 & -1.209991 & -0.924789 & 0.577866 & 1 & 1 & 14 & 21 \\ \mathrm{C} & 14 & -1.214996 & -2.091705 & 1.307037 & 1 & 13 & 15 & 20 \\ \mathrm{C} & 15 & 0.000000 & -2.652267 & 1.657303 & 1 & 14 & 16 & 22 \\ \mathrm{C} & 16 & 1.214996 & -2.091705 & 1.307037 & 1 & 15 & 17 & 19 \\ \mathrm{C} & 17 & 1.209991 & -0.924789 & 0.577866 & 1 & 1 & 16 & 18 \\ \mathrm{H} & 18 & 2.159988 & -0.509247 & 0.318207 & 5 & 17 & & \\ \mathrm{H} & 19 & 2.130997 & -2.560669 & 1.600082 & 5 & 16 & & \\ \mathrm{H} & 20 & -2.130997 & -2.560669 & 1.600082 & 5 & 14 & & \\ \mathrm{H} & 21 & -2.159988 & -0.509247 & 0.318207 & 5 & 13 & & \\ \mathrm{~N} & 22 & 0.000000 & -3.899750 & 2.436813 & 8 & 15 & 23 & 24 \\ \mathrm{O} & 23 & 1.061996 & -4.350906 & 2.718735 & 6 & 22 & & \end{array}$


$\begin{array}{lllllll}\text { O } & 24 & -1.061996 & -4.350906 & 2.718735 & 6 & 22\end{array}$

$\begin{array}{ll}\mathrm{E}\left(\mathrm{HF} / 6-31 \mathrm{G}^{*}\right) & -737.6972219 \\ \mathrm{E}\left(\mathrm{pBP} / \mathrm{DN}^{*} / / \mathrm{HF} / 6-\right. & \\ 31 \mathrm{G}^{*} & -742.355417 \\ \mathrm{E}(\mathrm{B} 3 \mathrm{LYP}) & -742.091452 \\ \mathrm{E}\left(\mathrm{BP} / 6-31 \mathrm{G}^{*}\right) & -742.102324\end{array}$

4-Hydroxy-4-phenyl -2,5-cyclohexadienone (4a)

24

$\begin{array}{lllllllll}\text { C } & 1 & -0.481003 & -2.301163 & 2.222198 & 1 & 2 & 5 & 6\end{array}$

$\begin{array}{lllllllll}\mathrm{C} & 2 & -0.082000 & -2.693710 & 0.848312 & 1 & 1 & 3 & 4\end{array}$

$\begin{array}{lllllllll}\text { C } & 3 & 0.558990 & -1.859390 & 0.048157 & 1 & 2 & 11 & 12\end{array}$

$\begin{array}{lllllll}\mathrm{H} & 4 & -0.341995 & -3.692000 & 0.547287 & 5 & 2\end{array}$

$\begin{array}{lllllll}\mathrm{O} & 5 & -1.080002 & -3.046402 & 2.941864 & 6 & 1\end{array}$

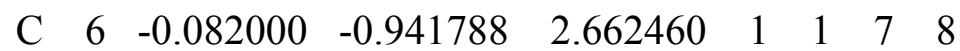

$\begin{array}{lllllllll}\text { C } & 7 & 0.558990 & -0.113022 & 1.856567 & 1 & 6 & 9 & 11\end{array}$

$\begin{array}{lllllll}\mathrm{H} & 8 & -0.341995 & -0.675797 & 3.670639 & 5 & 6\end{array}$

$\begin{array}{lllllll}\mathrm{H} & 9 & 0.852982 & 0.868515 & 2.184875 & 5 & 7\end{array}$

$\begin{array}{lllllllll}\text { C } & 10 & 0.295990 & 0.549576 & -0.530731 & 1 & 11 & 14 & 15\end{array}$

$\begin{array}{llllllllll}\text { C } & 11 & 0.946991 & -0.448135 & 0.432755 & 1 & 3 & 7 & 10 & 23\end{array}$

$\begin{array}{lllllll}\mathrm{H} & 12 & 0.852982 & -2.153244 & -0.944244 & 5 & 3\end{array}$

$\begin{array}{lllllllll}\text { C } & 13 & -0.927002 & 2.350815 & -2.270142 & 1 & 16 & 17 & 22\end{array}$

$\begin{array}{lllllllll}\mathrm{C} & 14 & 1.063980 & 1.380417 & -1.333054 & 1 & 10 & 17 & 18\end{array}$

$\begin{array}{lllllllll}\text { C } & 15 & -1.091003 & 0.628693 & -0.607132 & 1 & 10 & 16 & 19\end{array}$

$\begin{array}{lllllllll}\text { C } & 16 & -1.699005 & 1.522125 & -1.469894 & 1 & 13 & 15 & 20\end{array}$

$\begin{array}{lllllllll}\text { C } & 17 & 0.451981 & 2.276000 & -2.197906 & 1 & 13 & 14 & 21\end{array}$

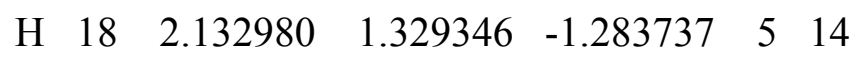

$\begin{array}{lllllll}\mathrm{H} & 19 & -1.701004 & -0.007919 & 0.007645 & 5 & 15\end{array}$

$\begin{array}{lllllll}\mathrm{H} & 20 & -2.772003 & 1.571045 & -1.517136 & 5 & 16\end{array}$

$\begin{array}{lllllll}\mathrm{H} & 21 & 1.058990 & 2.914764 & -2.814758 & 5 & 17\end{array}$

$\begin{array}{lllllll}\mathrm{H} & 22 & -1.397995 & 3.046402 & -2.941879 & 5 & 13\end{array}$

$\begin{array}{llllllll}\mathrm{O} & 23 & 2.344986 & -0.310745 & 0.300079 & 6 & 11 & 24\end{array}$

$\begin{array}{lllllll}\mathrm{H} & 24 & 2.771988 & -0.920029 & 0.888458 & 21 & 23\end{array}$

$\mathrm{E}\left(\mathrm{HF} / 6-31 \mathrm{G}^{*}\right) \quad-609.93427$

$\mathrm{E}(\mathrm{pBP} / \mathrm{DN} * / / \mathrm{HF} / 6-$

31G* $\quad-613.896097$

$\mathrm{E}\left(\mathrm{B} 3 \mathrm{LYP} / 6-31 \mathrm{G}^{*}\right) \quad-613.693777$

$\mathrm{E}\left(\mathrm{BP} / 6-31 \mathrm{G}^{*}\right) \quad-613.680931$

4-Hydroxy-4-(4'-methoxyphenyl)- 2,5-cyclohexadienone (4b) 28

$\begin{array}{lrrrrrrrrr}\mathrm{C} & 1 & -4.371490 & 0.914978 & 0.000000 & 1 & 2 & 7 & 23 & \\ \mathrm{C} & 2 & -3.750488 & 0.439987 & -1.260986 & 1 & 1 & 3 & 22 & \\ \mathrm{C} & 3 & -2.680481 & -0.336000 & -1.255997 & 1 & 2 & 4 & 5 & \\ \mathrm{H} & 4 & -2.241486 & -0.686005 & -2.173996 & 5 & 3 & & & \\ \mathrm{C} & 5 & -1.982483 & -0.809998 & 0.000000 & 1 & 3 & 6 & 10 & 12 \\ \mathrm{C} & 6 & -2.680481 & -0.336000 & 1.255997 & 1 & 5 & 7 & 9 & \\ \mathrm{C} & 7 & -3.750488 & 0.439987 & 1.260986 & 1 & 1 & 6 & 8 & \\ \mathrm{H} & 8 & -4.224487 & 0.758987 & 2.170990 & 5 & 7 & & & \end{array}$




$\begin{array}{lcrrrrrrrr}\mathrm{H} & 9 & -2.241486 & -0.686005 & 2.173996 & 5 & 6 & & \\ \mathrm{O} & 10 & -1.956482 & -2.220993 & 0.000000 & 6 & 5 & 11 & & \\ \mathrm{H} & 11 & -2.848480 & -2.544998 & 0.000000 & 21 & 10 & & \\ \mathrm{C} & 12 & -0.529480 & -0.328995 & 0.000000 & 1 & 5 & 13 & 17 & \\ \mathrm{C} & 13 & -0.243500 & 1.037979 & 0.000000 & 1 & 12 & 14 & 21 & \\ \mathrm{C} & 14 & 1.052505 & 1.492981 & 0.000000 & 1 & 13 & 15 & 20 & \\ \mathrm{C} & 15 & 2.113495 & 0.588989 & 0.000000 & 1 & 14 & 16 & 24 & \\ \mathrm{C} & 16 & 1.846497 & -0.768005 & 0.000000 & 1 & 15 & 17 & 19 & \\ \mathrm{C} & 17 & 0.528503 & -1.216003 & 0.000000 & 1 & 12 & 16 & 18 & \\ \mathrm{H} & 18 & 0.338500 & -2.270004 & 0.000000 & 5 & 17 & & & \\ \mathrm{H} & 19 & 2.638504 & -1.490997 & 0.000000 & 5 & 16 & & & \\ \mathrm{H} & 20 & 1.271500 & 2.544983 & 0.000000 & 5 & 14 & & & \\ \mathrm{H} & 21 & -1.045486 & 1.753983 & 0.000000 & 5 & 13 & & & \\ \mathrm{H} & 22 & -4.224487 & 0.758987 & -2.170990 & 5 & 2 & & & \\ \mathrm{O} & 23 & -5.330490 & 1.630981 & 0.000000 & 6 & 1 & & & \\ \mathrm{O} & 24 & 3.346497 & 1.130981 & 0.000000 & 6 & 15 & 25 & & \\ \mathrm{C} & 25 & 4.471497 & 0.298981 & 0.000000 & 1 & 24 & 26 & 27 & 28 \\ \mathrm{H} & 26 & 5.330505 & 0.952988 & 0.000000 & 5 & 25 & & & \\ \mathrm{H} & 27 & 4.500504 & -0.328003 & -0.884995 & 5 & 25 & & & \\ \mathrm{H} & 28 & 4.500504 & -0.328003 & 0.884995 & 5 & 25 & & & \end{array}$

$\begin{array}{ll}\mathrm{E}\left(\mathrm{HF} / 6-31 \mathrm{G}^{*}\right) & -723.8149195 \\ \mathrm{E}\left(\mathrm{pBP} / \mathrm{DN}^{*} / / \mathrm{HF} / 6-\right. & \\ 31 \mathrm{G}^{*} & -728.465083 \\ \mathrm{E}\left(\mathrm{B} 3 \mathrm{LYP} / 6-31 \mathrm{G}^{*}\right) & -728.215927 \\ \mathrm{E}\left(\mathrm{BP} / 6-31 \mathrm{G}^{*}\right) & -728.203684\end{array}$

4-Hydroxy-4-(4'-methylphenyl)-2,5-cyclohexadienone (4c) 27

$\begin{array}{lccccccccc}\mathrm{C} & 1 & -3.827484 & 0.779007 & 0.912079 & 1 & 2 & 5 & 6 & \\ \mathrm{C} & 2 & -3.246490 & 1.397568 & -0.305313 & 1 & 1 & 3 & 4 & \\ \mathrm{C} & 3 & -2.241486 & 0.835892 & -0.955246 & 1 & 2 & 11 & 12 & \\ \mathrm{H} & 4 & -3.693481 & 2.320724 & -0.625626 & 5 & 2 & & & \\ \mathrm{O} & 5 & -4.724487 & 1.293365 & 1.514328 & 6 & 1 & & & \\ \mathrm{C} & 6 & -3.246490 & -0.520157 & 1.332596 & 1 & 1 & 7 & 8 & \\ \mathrm{C} & 7 & -2.241486 & -1.074234 & 0.676163 & 1 & 6 & 9 & 11 & \\ \mathrm{H} & 8 & -3.693481 & -0.980942 & 2.194290 & 5 & 6 & & & \\ \mathrm{H} & 9 & -1.832489 & -2.021667 & 0.980377 & 5 & 7 & & & \\ \mathrm{C} & 10 & -0.098511 & -0.228928 & -0.268036 & 1 & 11 & 14 & 15 & \\ \mathrm{C} & 11 & -1.586487 & -0.463379 & -0.542542 & 1 & 3 & 7 & 10 & 23 \\ \mathrm{H} & 12 & -1.832489 & 1.284546 & -1.843445 & 5 & 3 & & & \\ \mathrm{C} & 13 & 2.623489 & 0.229568 & 0.268784 & 1 & 16 & 17 & 22 & \\ \mathrm{C} & 14 & 0.883500 & -0.862152 & -1.009430 & 1 & 10 & 17 & 18 & \\ \mathrm{C} & 15 & 0.293503 & 0.639374 & 0.748596 & 1 & 10 & 16 & 19 & \\ \mathrm{C} & 16 & 1.628494 & 0.863434 & 1.010941 & 1 & 13 & 15 & 20 & \\ \mathrm{C} & 17 & 2.226500 & -0.631592 & -0.739502 & 1 & 13 & 14 & 21 & \\ \mathrm{H} & 18 & 0.607498 & -1.534332 & -1.796448 & 5 & 14 & & & \\ \mathrm{H} & 19 & -0.449493 & 1.144653 & 1.340195 & 5 & 15 & & & \\ \mathrm{H} & 20 & 1.903503 & 1.539520 & 1.802521 & 5 & 16 & & & \\ \mathrm{H} & 21 & 2.970490 & -1.136871 & -1.331085 & 5 & 17 & & & \\ \mathrm{C} & 22 & 4.082489 & 0.483505 & 0.566116 & 1 & 13 & 25 & 26 & 27 \\ \mathrm{O} & 23 & -1.677490 & -1.377808 & -1.613205 & 6 & 11 & 24 & & \end{array}$




$\begin{array}{ccccccc}\mathrm{H} & 24 & -2.592484 & -1.539520 & -1.802536 & 21 & 23 \\ \mathrm{H} & 25 & 4.724503 & -0.083450 & -0.097702 & 5 & 22 \\ \mathrm{H} & 26 & 4.328491 & 0.203918 & 1.586060 & 5 & 22 \\ \mathrm{H} & 27 & 4.328491 & 1.534637 & 0.449524 & 5 & 22\end{array}$

$\begin{array}{ll}\mathrm{E}\left(\mathrm{HF} / 6-31 \mathrm{G}^{*}\right) & -648.971646 \\ \mathrm{E}(\mathrm{pBP} / \mathrm{DN} * / / \mathrm{HF} / 6- & \\ 31 \mathrm{G}^{*} & -653.22308 \\ \mathrm{E}\left(\mathrm{B} 3 \mathrm{LYP} / 6-31 \mathrm{G}^{*}\right) & -653.011792 \\ \mathrm{E}\left(\mathrm{BP} / 6-31 \mathrm{G}^{*}\right) & -652.99613\end{array}$

4-(4'-Bromophenyl)-4-hydroxy-2,5-cyclohexadienone (4d) 24

\begin{tabular}{|c|c|c|c|c|c|c|c|c|}
\hline $\mathrm{C}$ & 1 & -3.636490 & 0.821487 & 0.000000 & 1 & 2 & 7 & 24 \\
\hline $\mathrm{C}$ & 2 & -3.003494 & 0.362488 & -1.260986 & 1 & 1 & 3 & 23 \\
\hline $\mathrm{C}$ & 3 & -1.912491 & -0.383500 & -1.256989 & 1 & 2 & 4 & 5 \\
\hline $\mathrm{H}$ & 4 & -1.465485 & -0.723495 & -2.175995 & 5 & 3 & & \\
\hline $\mathrm{C}$ & 5 & -1.205490 & -0.841492 & 0.000000 & 1 & 3 & 6 & 10 \\
\hline $\mathrm{C}$ & 6 & -1.912491 & -0.383500 & 1.256989 & 1 & 5 & & 9 \\
\hline $\mathrm{C}$ & 7 & -3.003494 & 0.362488 & 1.260986 & 1 & 1 & 6 & 8 \\
\hline $\mathrm{H}$ & 8 & -3.486496 & 0.667496 & 2.170990 & 5 & 7 & & \\
\hline $\mathrm{H}$ & 9 & -1.465485 & -0.723495 & 2.175995 & 5 & 6 & & \\
\hline $\mathrm{O}$ & 10 & -1.139496 & -2.249496 & 0.000000 & 6 & 5 & 11 & \\
\hline $\mathrm{H}$ & 11 & -2.020493 & -2.600494 & 0.000000 & 21 & 10 & & \\
\hline $\mathrm{C}$ & 12 & 0.236496 & -0.322495 & 0.000000 & 1 & 5 & 13 & 17 \\
\hline $\mathrm{C}$ & 13 & 0.479492 & 1.047485 & 0.000000 & 1 & 12 & 14 & 22 \\
\hline $\mathrm{C}$ & 14 & 1.769500 & 1.542496 & 0.000000 & 1 & 13 & 15 & 21 \\
\hline $\mathrm{C}$ & 15 & 2.831497 & 0.654495 & 0.000000 & 1 & 14 & 16 & 20 \\
\hline $\mathrm{C}$ & 16 & 2.614502 & -0.708496 & 0.000000 & 1 & 15 & 17 & 19 \\
\hline $\mathrm{C}$ & 17 & 1.314500 & -1.192490 & 0.000000 & 1 & 12 & 16 & 18 \\
\hline $\mathrm{H}$ & 18 & 1.146500 & -2.250488 & 0.000000 & 5 & 17 & & \\
\hline $\mathrm{H}$ & 19 & 3.443497 & -1.390488 & 0.000000 & 5 & 16 & & \\
\hline $\mathrm{Br}$ & 20 & 4.612503 & 1.324493 & 0.000000 & 13 & 15 & & \\
\hline $\mathrm{H}$ & 21 & 1.944489 & 2.600494 & 0.000000 & 5 & 14 & & \\
\hline $\mathrm{H}$ & 22 & -0.341507 & 1.740494 & 0.000000 & 5 & 13 & & \\
\hline $\mathrm{H}$ & 23 & -3.486496 & 0.667496 & -2.170990 & 5 & 2 & & \\
\hline $\mathrm{O}$ & 24 & -4.612488 & 1.512497 & 0.000000 & 6 & 1 & & \\
\hline
\end{tabular}

$\begin{array}{ll}\mathrm{E}\left(\mathrm{HF} / 6-31 \mathrm{G}^{*}\right) & -3181.455799 \\ \mathrm{E}(\mathrm{pBP} / \mathrm{DN} / / \mathrm{HF} / 6- & \\ 31 \mathrm{G}^{*} & -3187.796467 \\ \mathrm{E}\left(\mathrm{B} 3 \mathrm{LYP} / 6-31 \mathrm{G}^{*}\right) & -3186.97461 \\ \mathrm{E}\left(\mathrm{BP} / 6-31 \mathrm{G}^{*}\right) & -3187.21564\end{array}$

4-(4'-Cyanophenyl)-4-hydroxy-2,5-cyclohexadienone (4e) 25

$\begin{array}{lrrrrrrrrr}\mathrm{C} & 1 & -3.990982 & 1.034988 & 0.000000 & 1 & 2 & 7 & 23 & \\ \mathrm{C} & 2 & -3.387985 & 0.537994 & -1.261993 & 1 & 1 & 3 & 22 & \\ \mathrm{C} & 3 & -2.343979 & -0.272995 & -1.257996 & 1 & 2 & 4 & 5 & \\ \mathrm{H} & 4 & -1.918991 & -0.638992 & -2.175995 & 5 & 3 & & & \\ \mathrm{C} & 5 & -1.666977 & -0.771988 & 0.000000 & 1 & 3 & 6 & 10 & 12 \\ \mathrm{C} & 6 & -2.343979 & -0.272995 & 1.257996 & 1 & 5 & 7 & 9 & \end{array}$




$\begin{array}{ccccccccc}\mathrm{C} & 7 & -3.387985 & 0.537994 & 1.261993 & 1 & 1 & 6 & 8 \\ \mathrm{H} & 8 & -3.851990 & 0.869995 & 2.171997 & 5 & 7 & & \\ \mathrm{H} & 9 & -1.918991 & -0.638992 & 2.175995 & 5 & 6 & & \\ \mathrm{O} & 10 & -1.680984 & -2.180984 & 0.000000 & 6 & 5 & 11 & \\ \mathrm{H} & 11 & -2.580978 & -2.481995 & 0.000000 & 21 & 10 & & \\ \mathrm{C} & 12 & -0.197006 & -0.336990 & 0.000000 & 1 & 5 & 13 & 17 \\ \mathrm{C} & 13 & 0.121002 & 1.017990 & 0.000000 & 1 & 12 & 14 & 21 \\ \mathrm{C} & 14 & 1.434998 & 1.433990 & 0.000000 & 1 & 13 & 15 & 20 \\ \mathrm{C} & 15 & 2.457000 & 0.489990 & 0.000000 & 1 & 14 & 16 & 24 \\ \mathrm{C} & 16 & 2.149994 & -0.861984 & 0.000000 & 1 & 15 & 17 & 19 \\ \mathrm{C} & 17 & 0.826996 & -1.272995 & 0.000000 & 1 & 12 & 16 & 18 \\ \mathrm{H} & 18 & 0.595000 & -2.317993 & 0.000000 & 5 & 17 & & \\ \mathrm{H} & 19 & 2.938995 & -1.589996 & 0.000000 & 5 & 16 & & \\ \mathrm{H} & 20 & 1.673004 & 2.481995 & 0.000000 & 5 & 14 & & \\ \mathrm{H} & 21 & -0.662003 & 1.754990 & 0.000000 & 5 & 13 & & \\ \mathrm{H} & 22 & -3.851990 & 0.869995 & -2.171997 & 5 & 2 & & \\ \mathrm{O} & 23 & -4.921982 & 1.785995 & 0.000000 & 6 & 1 & & \\ \mathrm{C} & 24 & 3.837006 & 0.918991 & 0.000000 & 1 & 15 & 25 & \\ \mathrm{~N} & 25 & 4.921997 & 1.255997 & 0.000000 & 8 & 24 & & \end{array}$

$\begin{array}{ll}\mathrm{E}\left(\mathrm{HF} / 6-31 \mathrm{G}^{*}\right) & -701.6689897 \\ \mathrm{E}(\mathrm{pBP} / \mathrm{DN} / / \mathrm{HF} / 6- & \\ 31 \mathrm{G}^{*} & -706.173565 \\ \mathrm{E}\left(\mathrm{B} 3 \mathrm{LYP} / 6-31 \mathrm{G}^{*}\right) & -705.934753 \\ \mathrm{E}\left(\mathrm{BP} / 6-31 \mathrm{G}^{*}\right) & -705.92318\end{array}$

4-Hydroxy-4-(4'-nitrophenyl)-2,5-cyclohexadienone (4f) 26

\begin{tabular}{|c|c|c|c|c|c|c|c|c|}
\hline $\mathrm{C}$ & 1 & -3.861984 & 1.107986 & 0.000000 & 1 & 2 & 7 & 23 \\
\hline $\mathrm{C}$ & 2 & -3.267990 & 0.597992 & -1.261993 & 1 & 1 & 3 & 22 \\
\hline $\mathrm{C}$ & 3 & -2.239990 & -0.231995 & -1.257996 & 1 & 2 & 4 & 5 \\
\hline $\mathrm{H}$ & 4 & -1.822983 & -0.606995 & -2.176987 & 5 & 3 & & \\
\hline $\mathrm{C}$ & 5 & -1.573990 & -0.744995 & 0.000000 & 1 & 3 & 6 & 10 \\
\hline $\mathrm{C}$ & 6 & -2.239990 & -0.231995 & 1.257996 & & 5 & & 9 \\
\hline $\mathrm{C}$ & 7 & -3.267990 & 0.597992 & 1.261993 & 1 & & 6 & 8 \\
\hline $\mathrm{H}$ & 8 & -3.725983 & 0.939987 & 2.1719 & & 7 & & \\
\hline $\mathrm{H}$ & 9 & -1.822983 & -0.606995 & 2.176987 & 5 & 6 & & \\
\hline $\mathrm{O}$ & 10 & -1.615982 & -2.153000 & 0.000000 & 6 & 5 & 11 & \\
\hline $\mathrm{H}$ & 11 & -2.521988 & -2.438004 & 0.000000 & 21 & 10 & & \\
\hline $\mathrm{C}$ & 12 & -0.096008 & -0.341003 & 0.000000 & 1 & 5 & 13 & 17 \\
\hline $\mathrm{C}$ & 13 & 0.246994 & 1.00 & 0.000000 & 1 & 12 & 14 & 21 \\
\hline $\mathrm{C}$ & 14 & 1.569000 & 1.40 & 0.0 & 1 & 13 & 15 & 20 \\
\hline $\mathrm{C}$ & 15 & 2.550995 & 0.42 & 0.000000 & 1 & 14 & 16 & 24 \\
\hline $\mathrm{C}$ & 16 & 2.240005 & -0.916992 & 0.000000 & 1 & 15 & 17 & 19 \\
\hline $\mathrm{C}$ & 17 & 0.908005 & -1.297989 & 0.000000 & 1 & 12 & 16 & 18 \\
\hline $\mathrm{H}$ & 18 & 0.655000 & -2.3 & 0.000000 & 5 & 17 & & \\
\hline $\mathrm{H}$ & 19 & 3.022003 & -1.649002 & 0.000000 & 5 & 16 & & \\
\hline $\mathrm{H}$ & 20 & 1.839996 & 2.437988 & 0.000000 & 5 & 14 & & \\
\hline $\mathrm{H}$ & 21 & -0.519989 & 1.760986 & 0.000000 & 5 & 13 & & \\
\hline $\mathrm{H}$ & 22 & -3.725983 & 0.939987 & -2.171997 & 5 & 2 & & \\
\hline $\mathrm{O}$ & 23 & -4.777985 & 1.875992 & 0.000000 & 6 & 1 & & \\
\hline $\mathrm{N}$ & 24 & 3.951004 & 0.830994 & 0.000000 & 8 & 15 & 25 & 26 \\
\hline
\end{tabular}


$\begin{array}{lllllll}\mathrm{O} & 25 & 4.778000 & -0.030000 & 0.000000 & 6 & 24\end{array}$

$\begin{array}{lllllll}\mathrm{O} & 26 & 4.188995 & 2.000992 & 0.000000 & 6 & 24\end{array}$

$\mathrm{E}\left(\mathrm{HF} / 6-31 \mathrm{G}^{*}\right)$

$-813.4052177$

$\mathrm{E}(\mathrm{pBP} / \mathrm{DN} * / / \mathrm{HF} / 6-31 \mathrm{G} *$

$-818.508878$

$\mathrm{E}\left(\mathrm{B} 3 \mathrm{LYP} / 6-31 \mathrm{G}^{*}\right)$

$-818.190263$

$\mathrm{E}\left(\mathrm{BP} / 6-31 \mathrm{G}^{*}\right)$

$-818.20159$

Comparisons of selected HF/6-31G* bond lengths in 1a-f

\begin{tabular}{|l|l|l|l|l|}
\hline Ion & $\begin{array}{l}\text { C1-C2 bond } \\
\text { length }(\AA)\end{array}$ & $\begin{array}{l}\text { C2-C3 bond } \\
\text { length }(\AA)\end{array}$ & $\begin{array}{l}\text { C3-C4 bond } \\
\text { length }(\AA)\end{array}$ & $\begin{array}{l}\text { C4-O bond } \\
\text { length }(\AA)\end{array}$ \\
\hline 1a & 1.460 & 1.331 & 1.486 & 1.188 \\
\hline 1b & 1.467 & 1.329 & 1.485 & 1.189 \\
\hline 1c & 1.462 & 1.330 & 1.485 & 1.188 \\
\hline 1d & 1.460 & 1.332 & 1.486 & 1.187 \\
\hline 1e & 1.456 & 1.333 & 1.486 & 1.187 \\
\hline 1f & 1.454 & 1.344 & 1.486 & 1.187 \\
\hline
\end{tabular}


C13 NMR of 2c
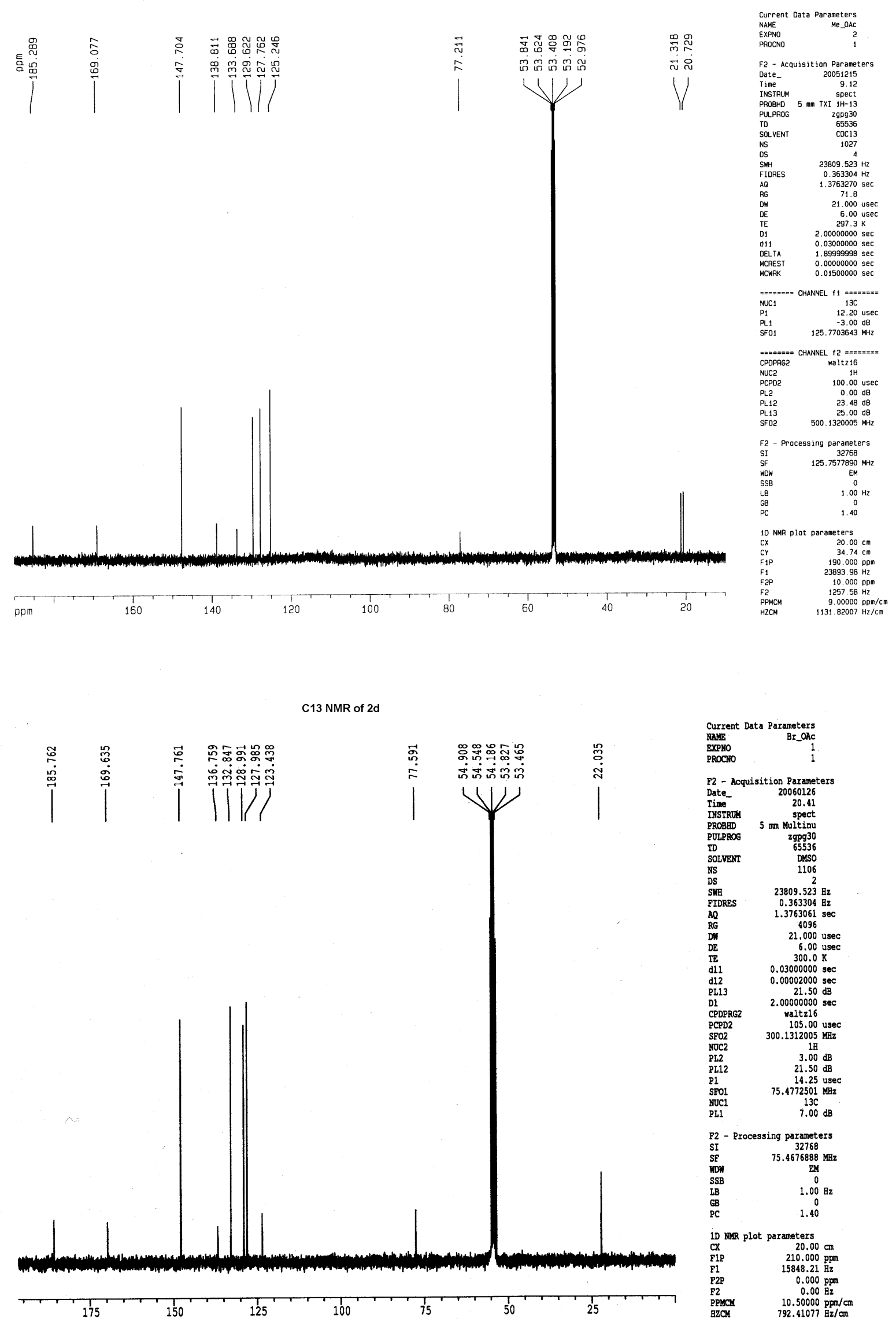

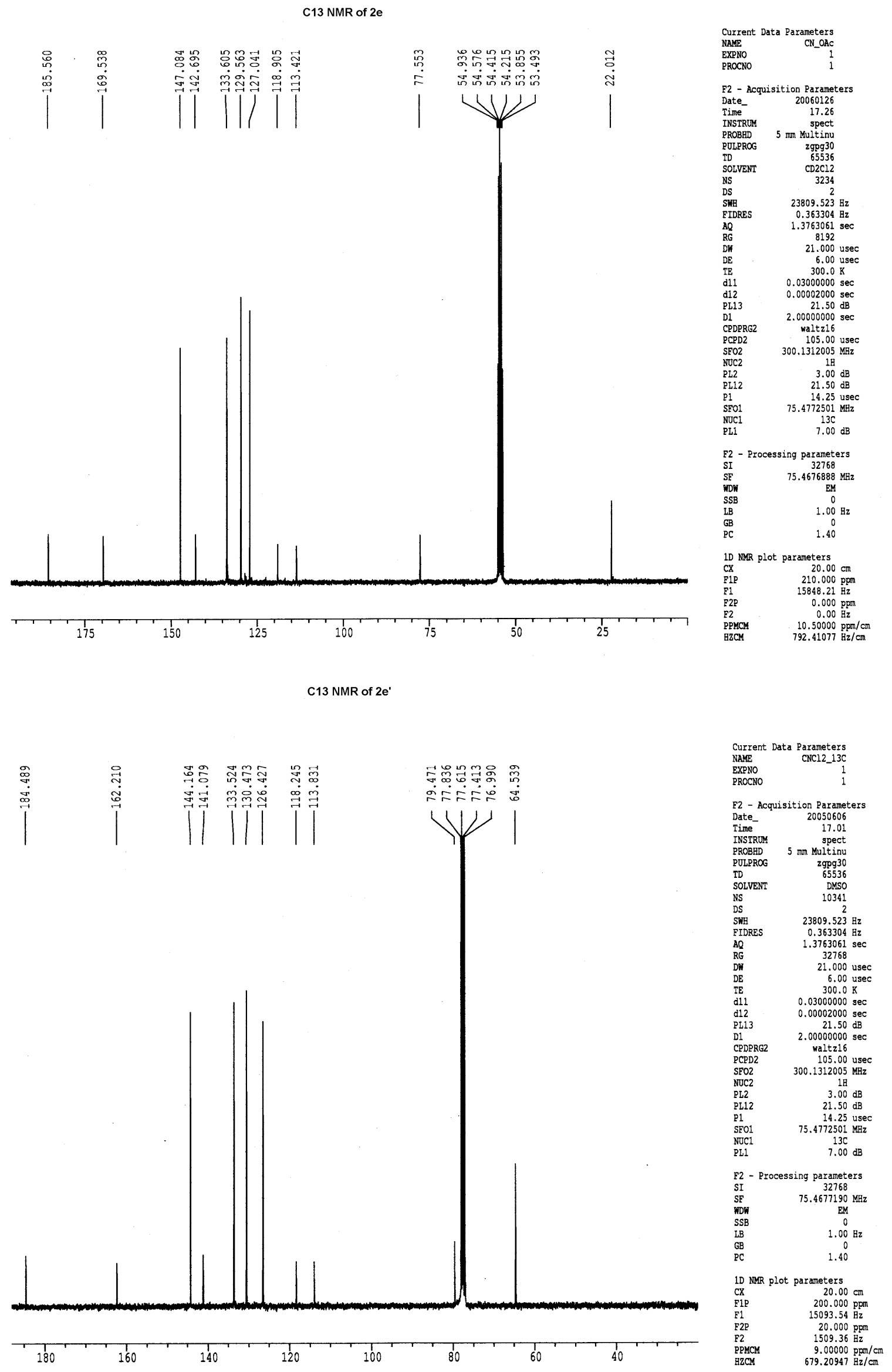
C13 NMR of $2 f^{\prime}$

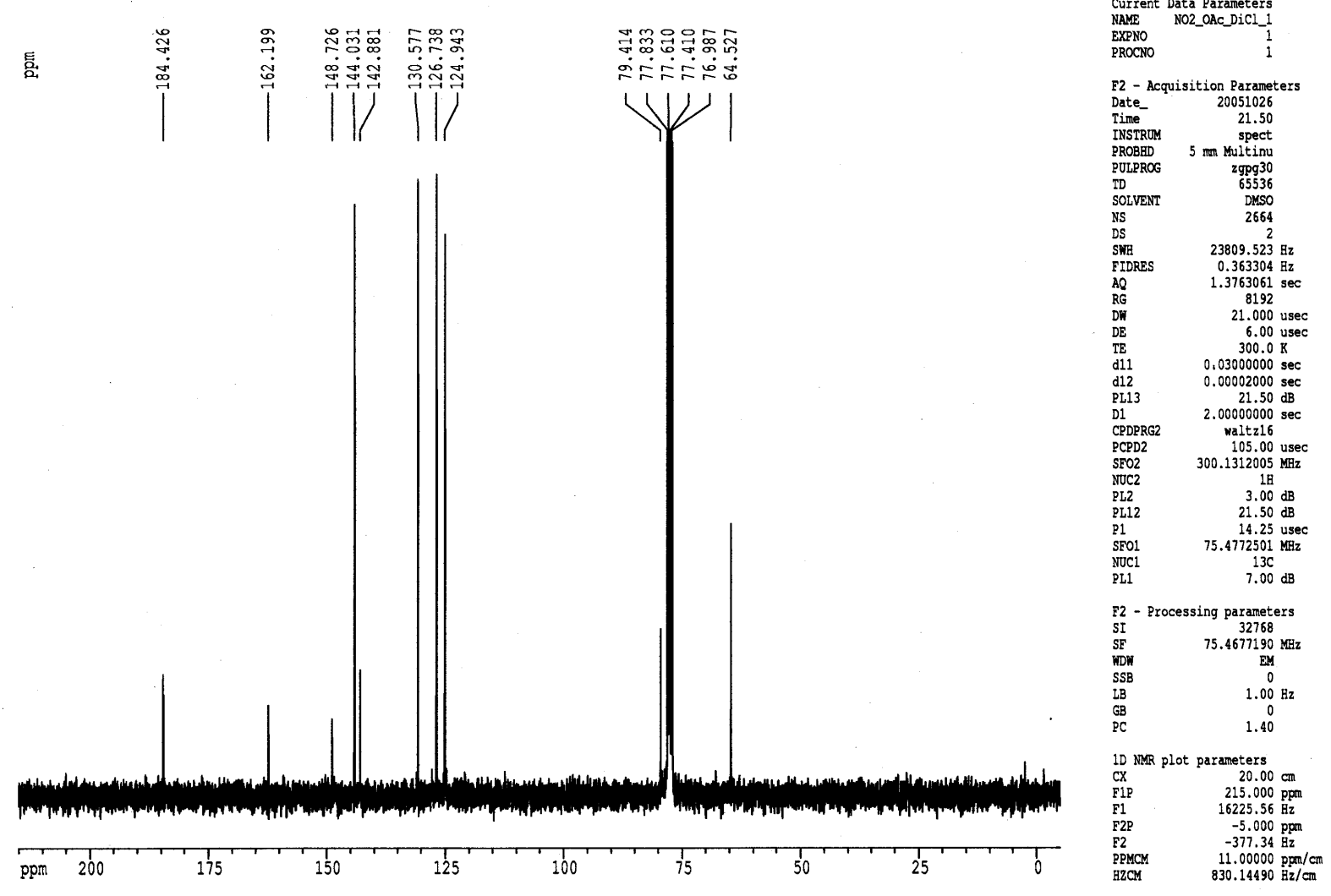

C13 NMR of $3 b$

\begin{tabular}{|c|c|c|}
\hline & 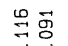 & 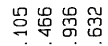 \\
\hline a & ㅁํㅁำ & 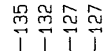 \\
\hline
\end{tabular}

ญิ巛

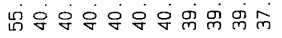

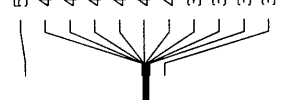

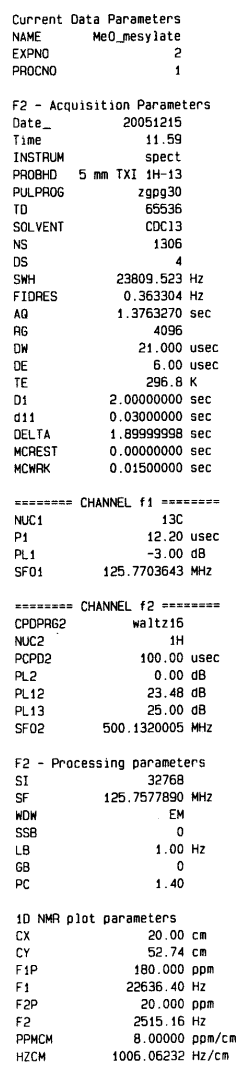




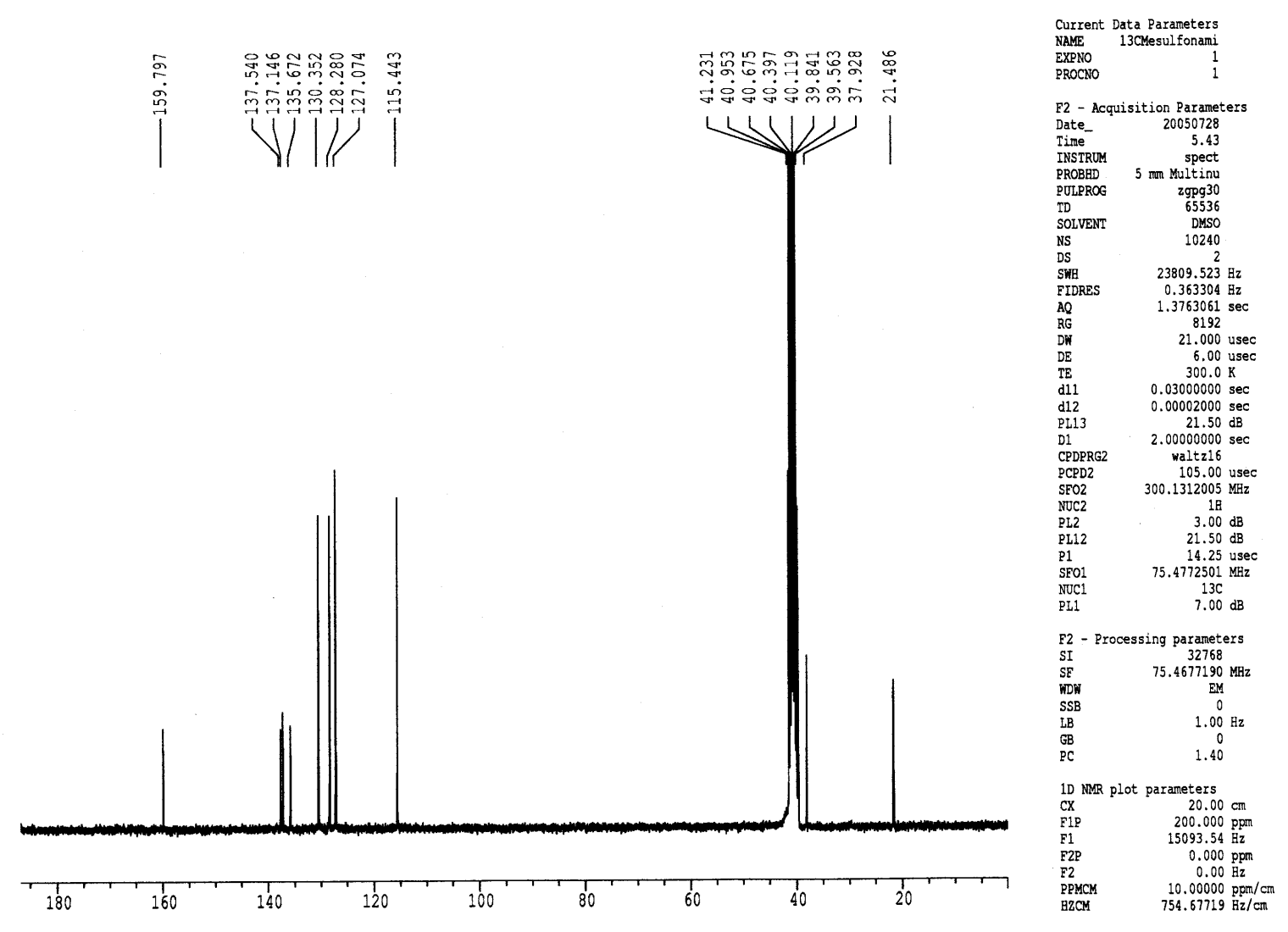

C13 NMR of 4b

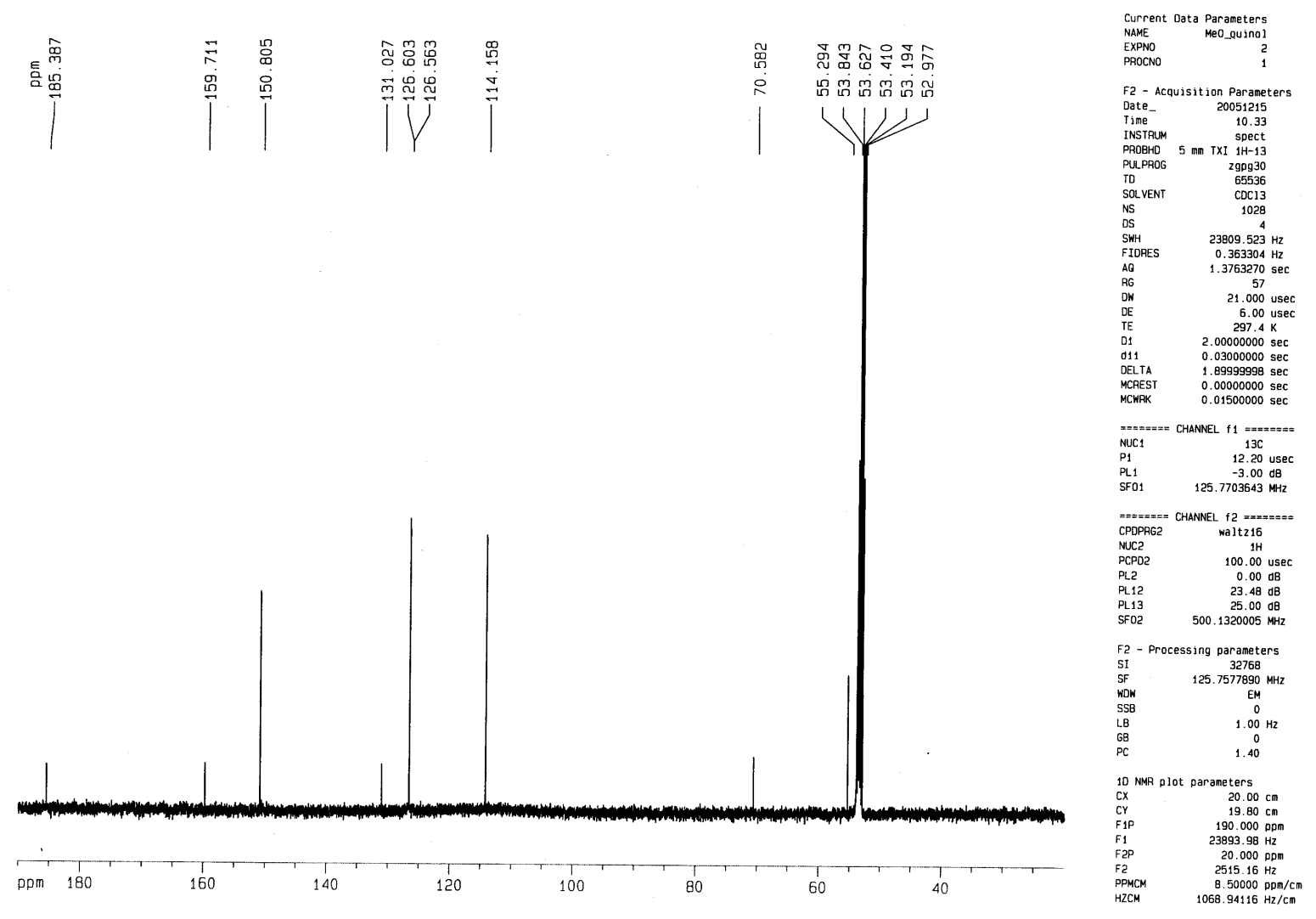




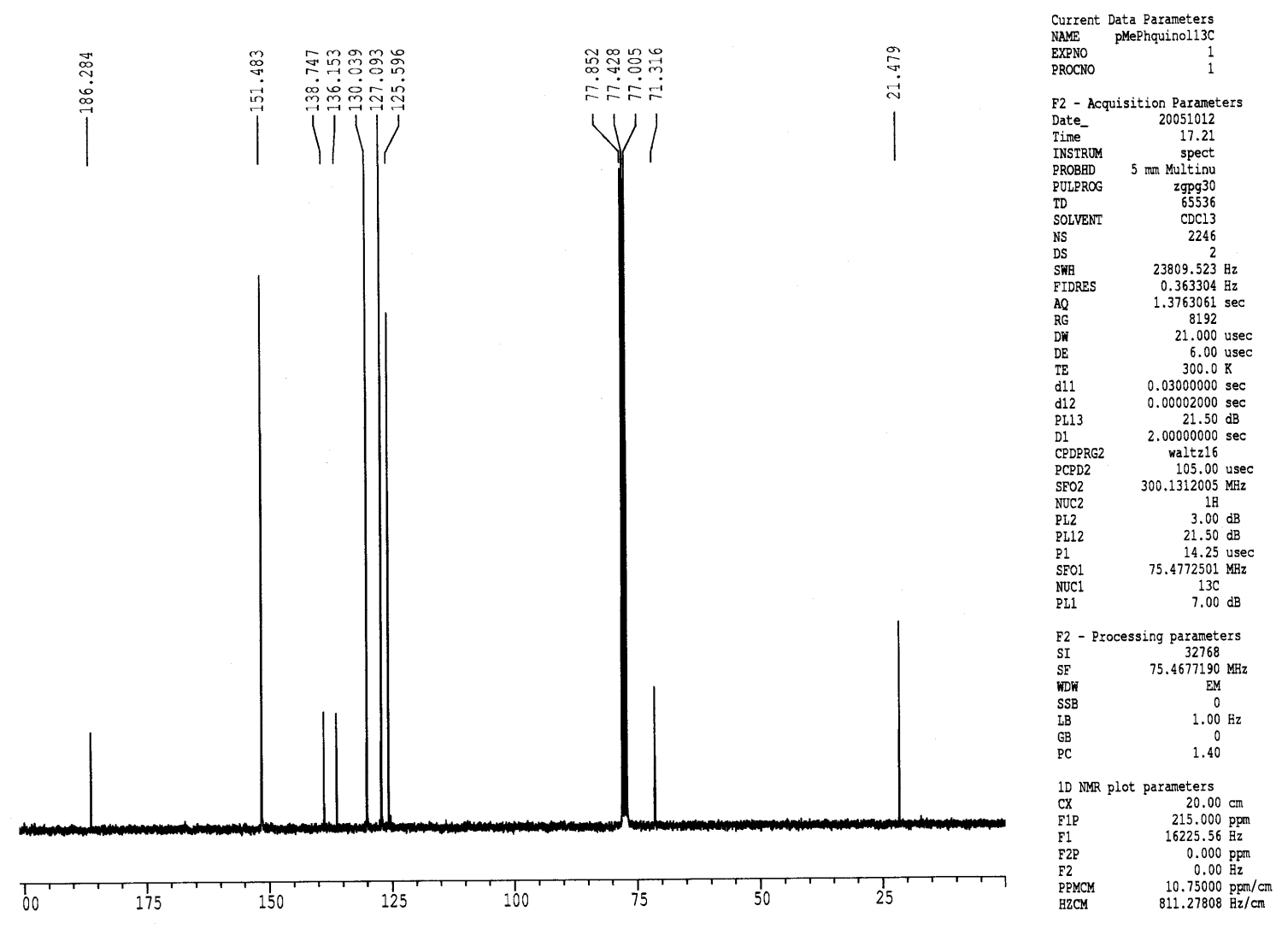

C13 NMR of 4d

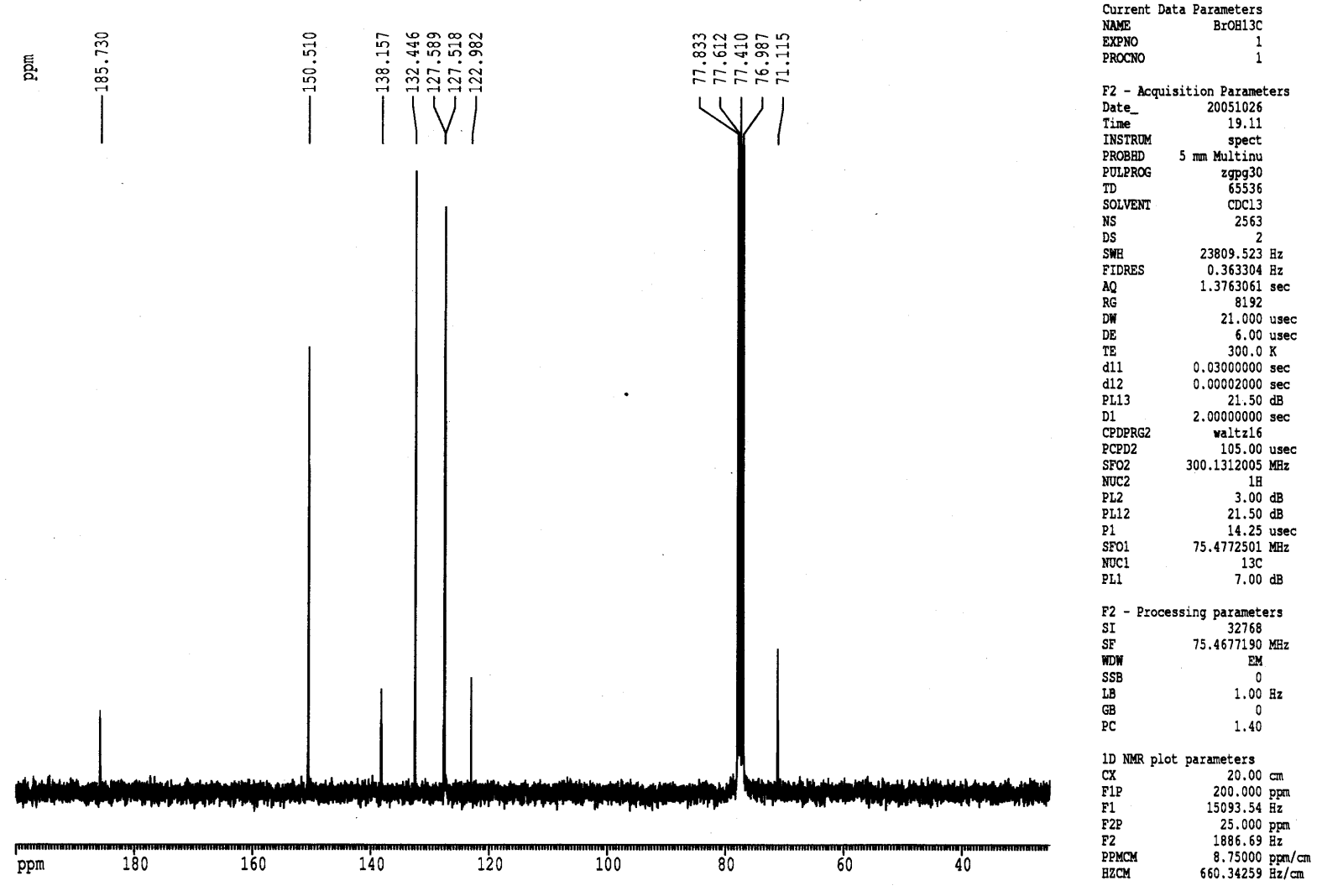


C13 NMR of $4 e$
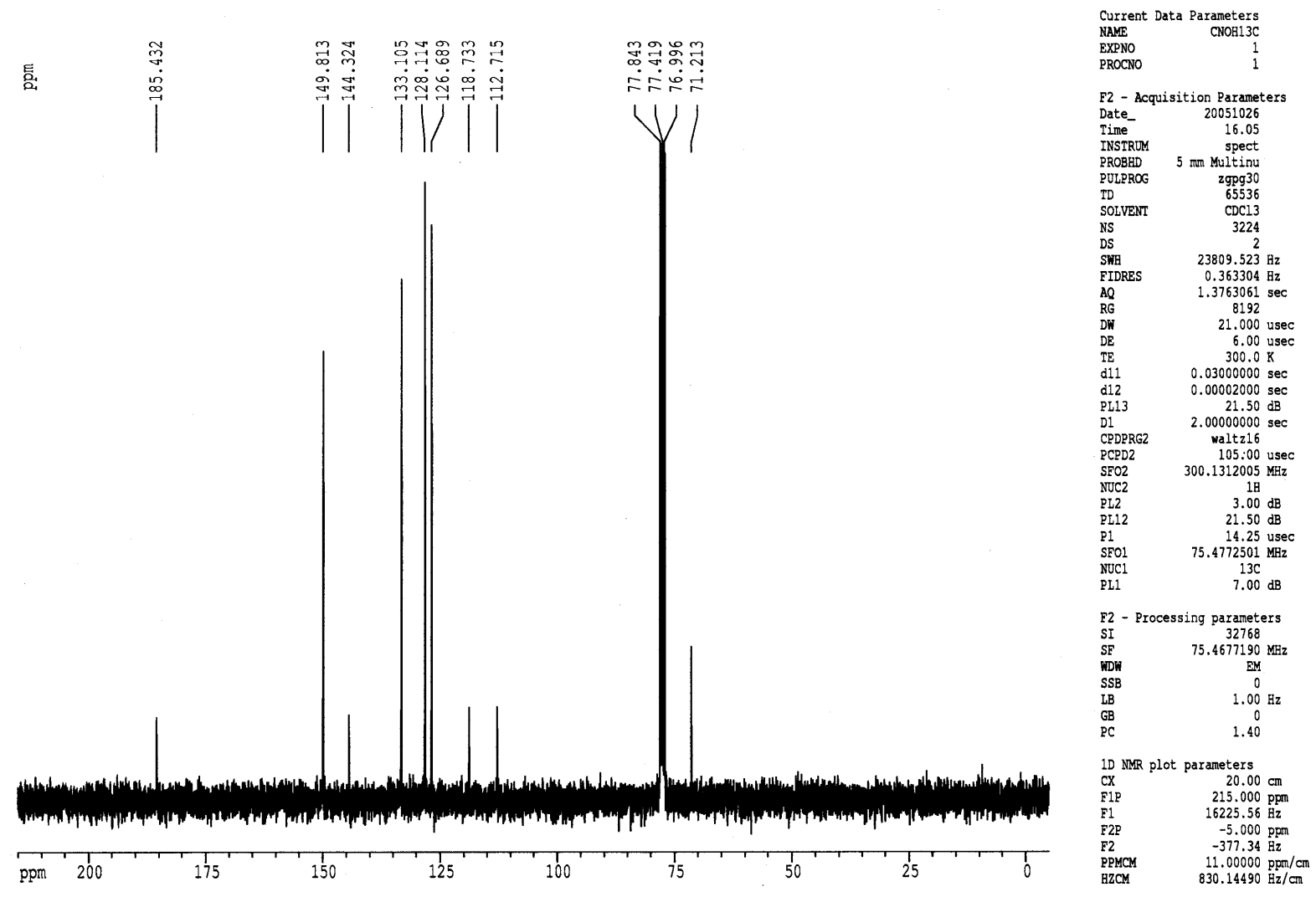

C13 NMR of $4 f$

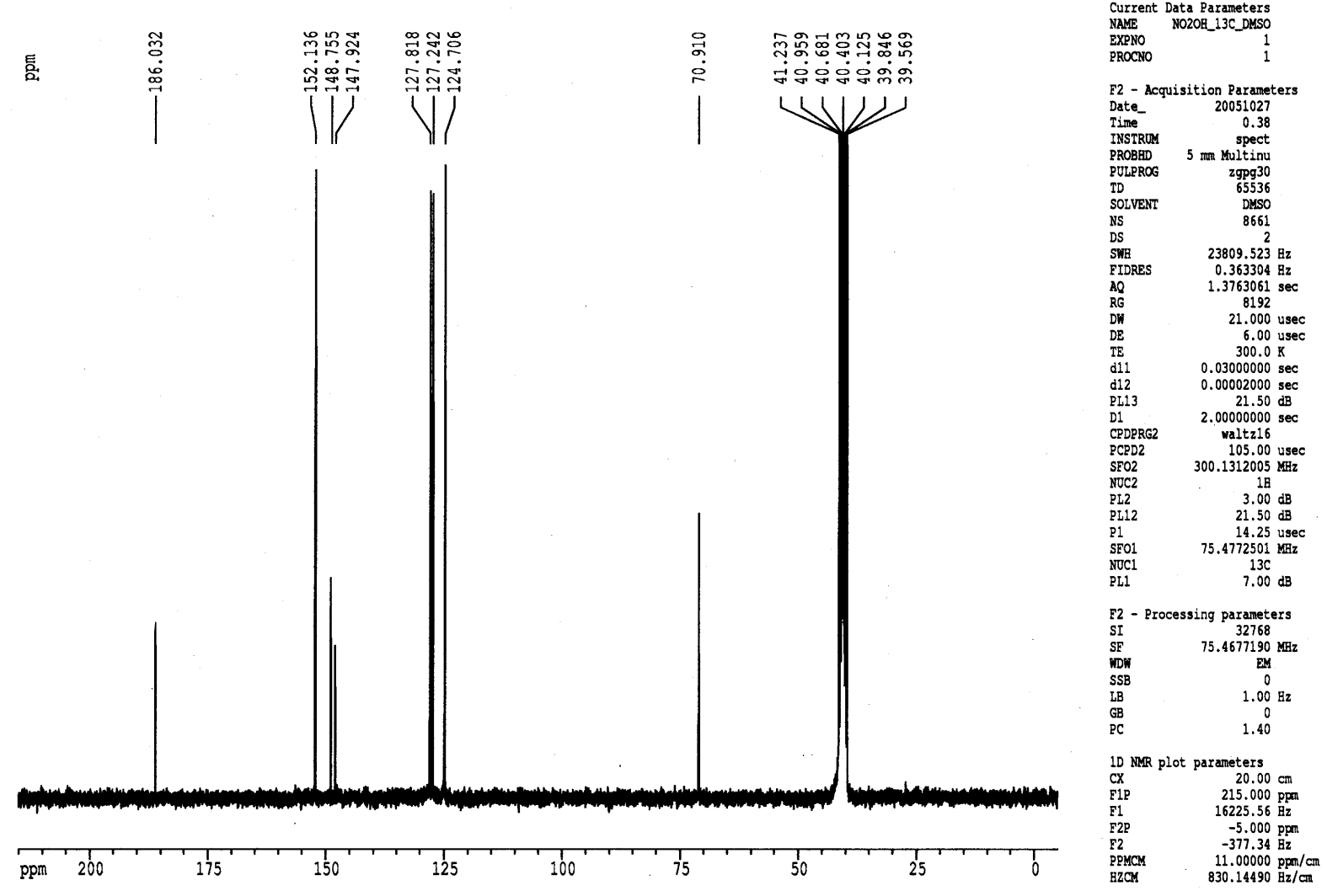




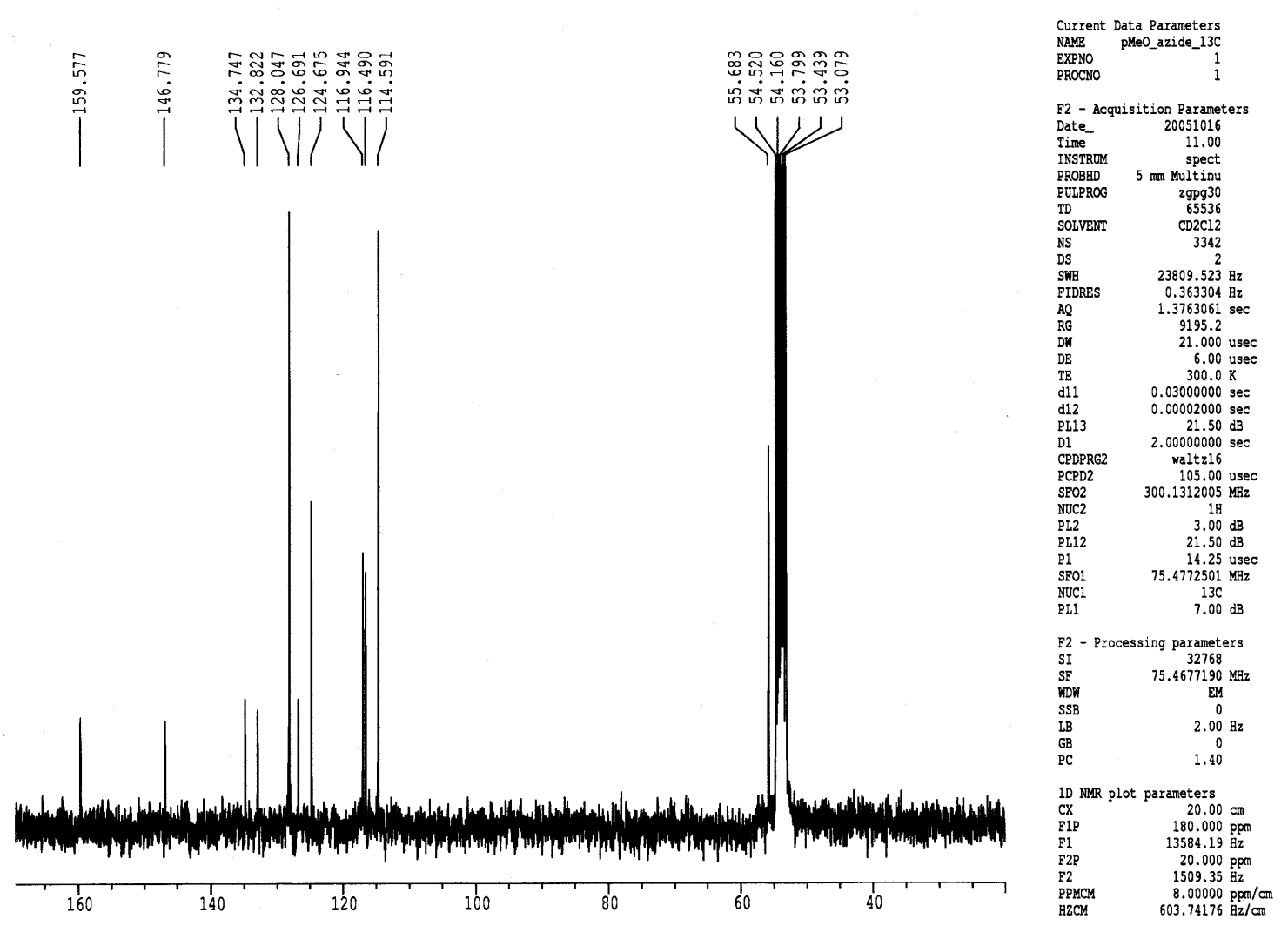

C13 NMR of 5c

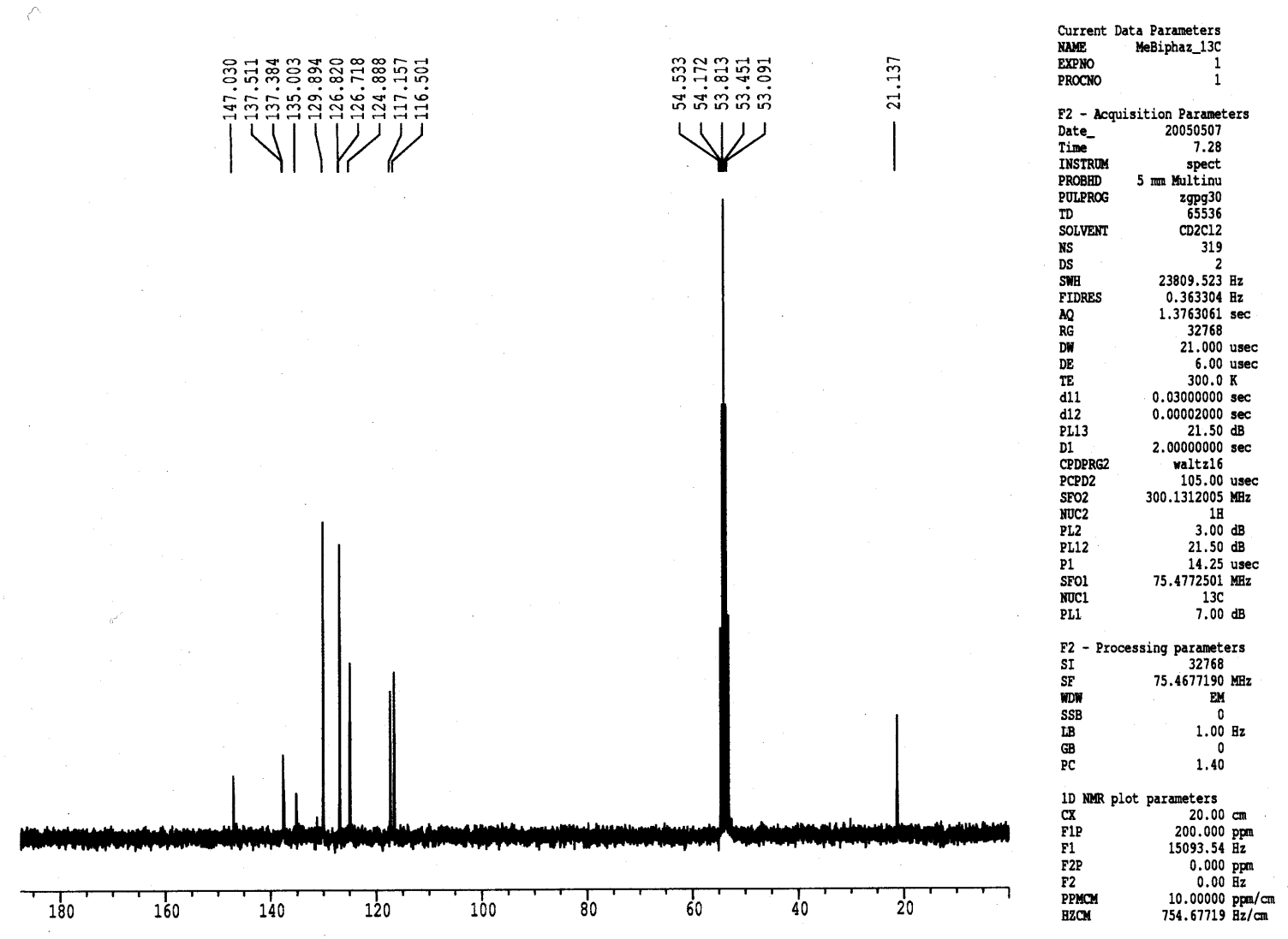



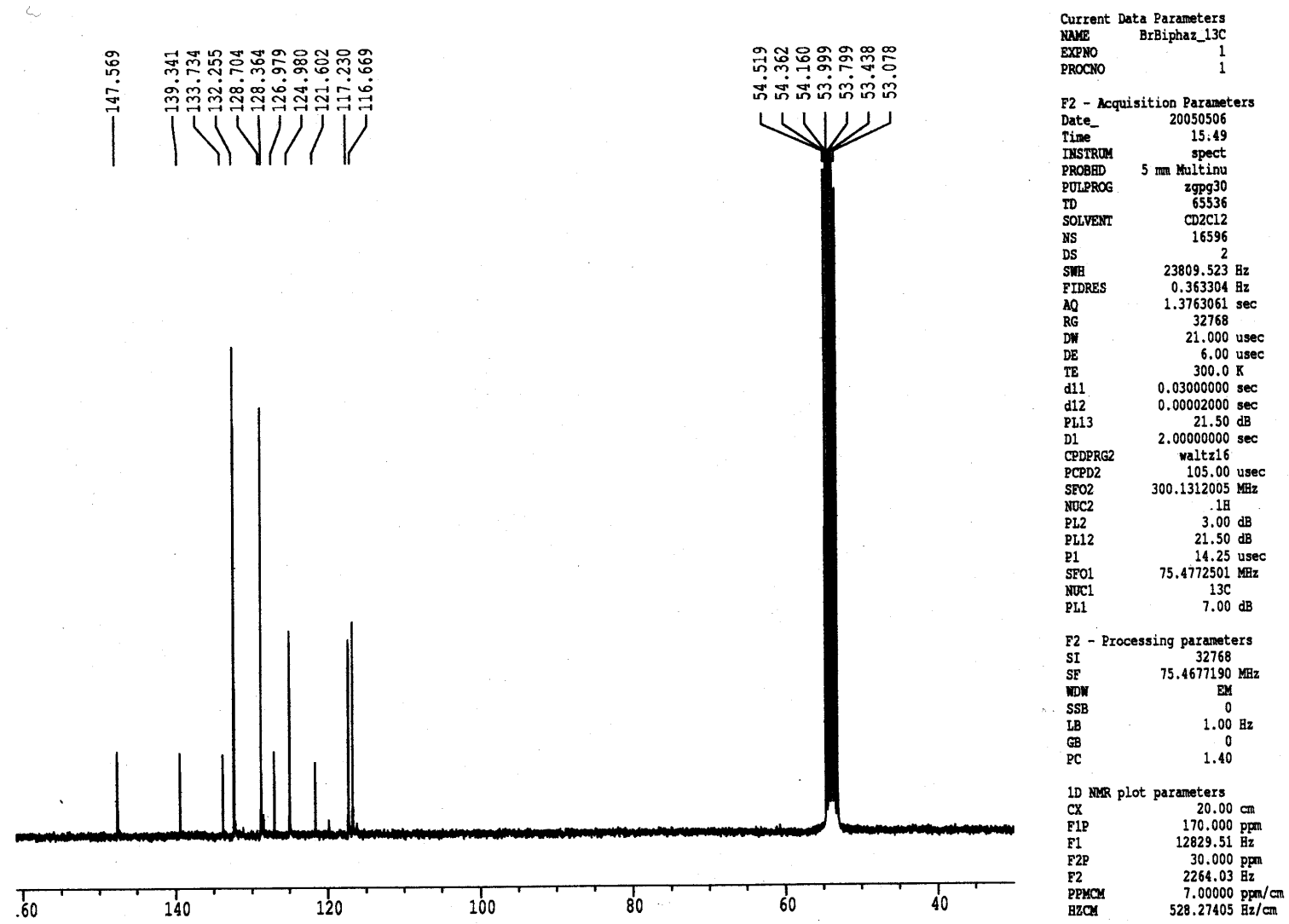

C13 NMR of $5 e$

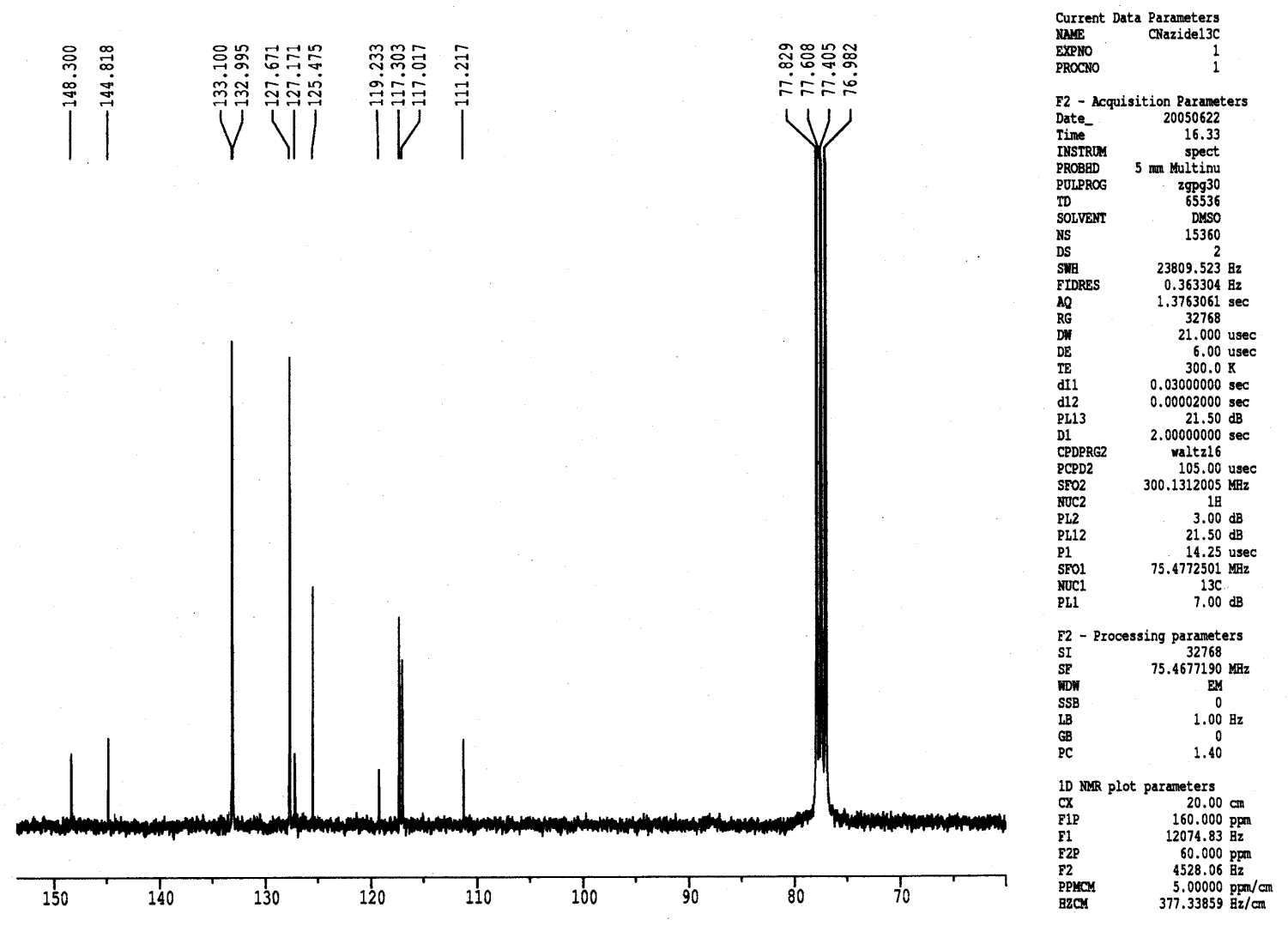



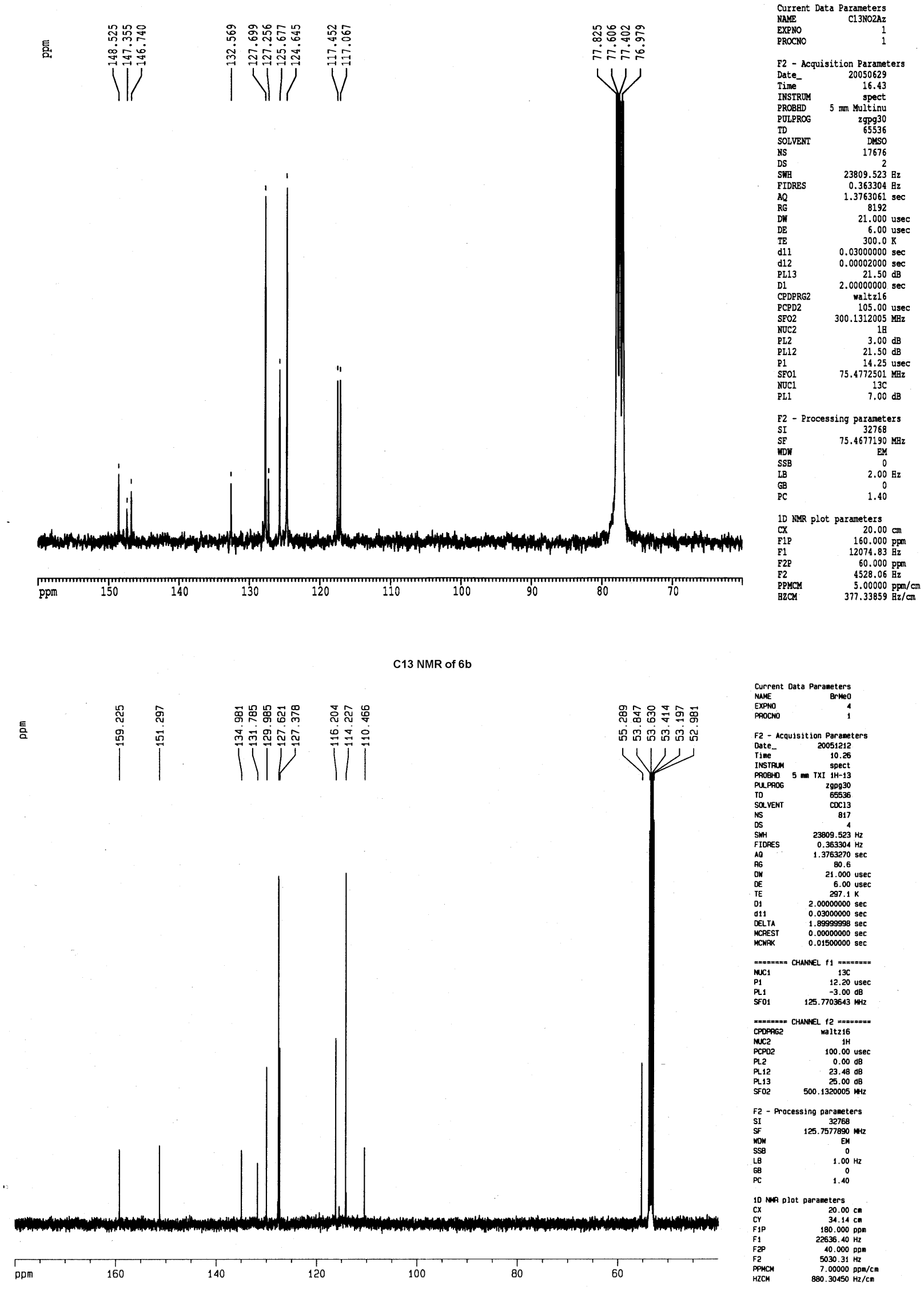